\title{
Comportamiento reológico del aluminio AA6061 por efecto de las transformaciones de fase activadas térmicamente
}

\author{
Rheological behavior of the AA6061 aluminum \\ by effect of the thermally activated phase \\ transformations
}

José Luis Ochoa-M.

\begin{abstract}
Laboratorio de Medidas Eléctricas en Materiales, Centro de Ingeniería de Materiales y Nanotecnología, Instituto Venezolano de Investigaciones Científicas, IVIC. Apartado Postal 21827, Caracas 1020A - Venezuela.

E-mail: jlochoa@ivic.gob.ve
\end{abstract}

\section{RESUMEN}

Las aleaciones de aluminio a la temperatura ambiente no experimentan comportamientos reológicos apreciables debido a su orden microestructural coherente y su alta compacidad. Sin embargo, el aumento de la temperatura genera campos de tensiones, y con ello deformaciones, que alteran el equilibrio microestructural, manifestándose el comportamiento reológico como consecuencia de la fricción interna. En las aleaciones de Al-Mg-Si la precipitación de fases provoca pérdida de coherencia produciendo fricción interna. Los objetivos de este trabajo fueron caracterizar e identificar las transformaciones microestructurales experimentadas en el aluminio AA6061 por efecto de la temperatura; estudiar la cinética no-isotérmica e isotérmica, a través de modelos atomísticos. Analizar el comportamiento reológico, manifestado en la deformación, y en la relajación de tensiones, considerando los postulados expresados por el Número de Deborah $\left(\mathrm{D}_{\mathrm{e}}\right)$. El desarrollo experimental ha sido orientado y organizado a través de la resistividad eléctrica y la microscopía. Cuatro regiones de transformaciones fueron observadas: I: $20-190$, II: $220-420$, III: $420-520$, IV: $\geq 525^{\circ} \mathrm{C}$; con energías de activación: $E_{\mathrm{a}}^{\mathrm{I}}: 20-60, \mathrm{E}_{\mathrm{a}}^{\mathrm{II}}: 90-120, \mathrm{E}_{\mathrm{a}}^{\mathrm{III}}: 400-470 \mathrm{~kJ} / \mathrm{mol}$; y conformadas de agregados de soluto y fases aciculares y cilíndricas, respectivamente. Se determinó que $\mathrm{D}_{\mathrm{e}} \leq 1$, asociándose a un comportamiento viscoelástico. Se observó, entre 100 y $200{ }^{\circ} \mathrm{C}$, que: $0,1 \leq \mathrm{D}_{\mathrm{e}} \leq 0,3$, vinculándose a un considerable nivel de difusión, con tasa de nucleación máxima a $150{ }^{\circ} \mathrm{C}$. Estas caracterizaciones ampliarán el entendimiento del fenómeno de difusión, los procesos de precipitación, deformación y relajación; para aplicaciones prácticas y desarrollos teóricos relacionados.

Palabras clave: aluminio AA6061, comportamiento reológico, Número de Deborah, resistividad eléctrica, transformaciones de fase.

\section{ABSTRACT}

Aluminum alloys at room temperature not experience significant rheological behavior due to its coherent microstructural order, and its high compactness. However, the increase of the temperature generates stress fields, and thereby deformations, that alter the microstructural equilibrium, manifesting the rheological behavior as consequence of the internal friction. In the alloys of $\mathrm{Al}-\mathrm{Mg}$-Si, the precipitation of phases cause loss of coherence producing internal friction. The objectives of this work were characterize and identify the microstructural transformations experienced in the AA6061 aluminum by temperature effect; study the kinetic no-isothermal and isothermal, through atomistic models. Analyze the rheological behavior, manifested in the deformation, and in the stress relaxation, considering the postulates expressed by Deborah`s Number $\left(D_{e}\right)$. The experimental development has been oriented and organized through the electrical resistivity and microscopy. Four regions of transformations were observed: I: 20-190, II: 220-420, III: 420-520, IV: $\geq 525{ }^{\circ} \mathrm{C}$; with activation energys: $E_{a}^{\mathrm{I}}: 20-60, \mathrm{E}_{\mathrm{a}}^{\mathrm{II}}: 90-120, \mathrm{E}_{\mathrm{a}}^{\mathrm{III}}: 400-470 \mathrm{~kJ} / \mathrm{mol}$; and formed of solute aggregates and needle and cylindrical phases, respectively. It determined that $\mathrm{D}_{\mathrm{e}} \leq 1$, associated to a viscoelastic behavior. It observed, between 100 and $200{ }^{\circ} \mathrm{C}$, that: $0.1 \leq \mathrm{D}_{\mathrm{e}} \leq 0.3$, linking to a considerable degree of diffusion, with maximum rate of nucleation at $150{ }^{\circ} \mathrm{C}$. These characterizations will broaden the understanding of the diffu- 
sion phenomenon, the precipitation processes, deformation and relaxation; for practical applications and related theoretical developments.

Keywords: aluminum AA6061, Deborah`s Number, electrical resistivity, phase transformations, rheological behavior.

\section{INTRODUCCIÓN}

Las aleaciones de aluminio, y los metales en general, a la temperatura ambiente no experimentan comportamientos reológicos apreciables debido a su ordenamiento microestructural coherente y a su alta compacidad. Sin embargo, cuando aumenta la temperatura el nivel térmico crece generándose una serie de fenómenos que alteran el equilibrio microestructural, lo que conlleva a que se ponga de manifiesto el comportamiento reológico. El aumento de la temperatura da lugar a campos de tensiones, y con ello a deformaciones térmicas.

En los metales, el estudio del comportamiento reológico viscoelástico como consecuencia de la fricción interna tiene muy pocas investigaciones publicadas hasta el presente [1-3]. La fricción interna está esencialmente conectada al movimiento de las dislocaciones; este movimiento es alto en los metales puros y livianos como el aluminio, y bajo en los aceros [4]. La fricción interna también está relacionada directamente con el número y tipo de unidades microestructurales móviles (heterogeneidades), y con el movimiento específico que experimentan en el volumen del sólido, este movimiento es activado térmicamente y se encuadra de acuerdo a la ley de Arrhenius [5].

En ausencia de microesfuerzos (tensiones) la microestructura permanece en un estado de equilibrio que corresponde a un mínimo local de energía. En general, cuando se induce un microesfuerzo se promueve el movimiento de las dislocaciones lo que conduce a un nuevo estado de equilibrio. Este proceso da lugar a una deformación anelástica asociada al movimiento de las heterogeneidades entre ambas posiciones de equilibrio. Esta deformación estará desfasada respecto al microesfuerzo, siendo este desfasaje una medida de la fricción interna. Otro factor ya demostrado está relacionado con la pérdida de coherencia con la matriz que produce fricción interna, y está asociado, principalmente, con las partículas (fases secundarias) semicoherentes y no-coherentes con la matriz [6-13].

En las aleaciones de aluminio de la serie AA6000 (sistema: Al-Mg-Si), entre otras, el proceso de precipitación se basa en la formación de fases intermetálicas semicoherentes y no-coherentes que son producidas por descomposición de una solución sólida sobresaturada obtenida por tratamiento térmico de solución y temple. De esta forma, la aleación de aluminio AA6061 se endurece por precipitación conllevando a una mejor relación de valores de resistencia/ductilidad, siendo esta aleación muy apropiada para aplicaciones en los sectores aeroespacial, transporte, eléctrico, electromecánica, aplicaciones estructurales y fabricación de instrumentos domésticos y deportivos.

Basándose en estos estudios y aseveraciones, la investigación en este trabajo tiene como objetivos: La realización y la caracterización de las distintas transformaciones de fase producidas por efecto de la temperatura en la aleación de aluminio AA6061. El estudio de la cinética de estas transformaciones, en los regímenes no-isotérmico e isotérmico, mediante la implementación y aplicación del modelo atomístico de KJMAMITTEMEIJER [14-15]. Además, se estudia y analiza el comportamiento reológico experimentado por esta aleación de aluminio, y puesto de manifiesto a través de los procesos de deformación y de relajación de tensiones como consecuencias de estas transformaciones, y es interpretado mediante los postulados de Marcus Reiner expresados a través del Número de Deborah $\left(D_{e}\right)$ [16]. La investigación es basada mediante la orientación y organización experimental a través de la medida de la resistividad eléctrica, no-isotérmica e isotérmica, el empleo de la microscopía electrónica de transmisión y de barrido, y la determinación cualitativa y cuantitativa de fases por microanálisis elemental por rayos-X (EDX, por sus siglas en inglés). La implementación y aplicación de distintos modelos cuantitativos y la combinación de todas las técnicas utilizadas contribuirá a ampliar la comprensión del fenómeno de difusión y de los procesos de precipitación, transformación de fases, deformación y relajación en estas aleaciones, por lo cual ayudará al entendimiento (práctico y teórico) y las aplicaciones prácticas de estas aleaciones de aluminio.

\section{MATERIALES Y MÉTODOS}

\subsection{Material empleado, análisis de composición y tratamiento térmico de solución}

El material empleado en esta experiencia consistió de: una aleación de aluminio AA6061 proveniente de la empresa venezolana VENALUM, recibida en forma de tocho y con historial térmico desconocido (En Esstado de Entrega: EEE). También se empleó un aluminio comercial de alta pureza $7 \mathrm{~N}(7 \mathrm{~N}=7$ Nine $\equiv 99$, 
$99999 \%$, serie AA1000). Además, fueron empleados los datos experimentales de la resistividad eléctrica de un aluminio de pureza 99,995\%, reportados por SIMMONS y BALLUFFI [17].

La composición química de la aleación AA6061 fue determinada por análisis elemental a través de la Prueba de la Chispa Eléctrica Controlada siguiendo las normas ASTM E 101-67 y COVENIN 793-88, los ensayos se realizaron empleando un Espectrómetro de Lectura Directa, modelo M9, marca Spectrolab. La composición química del aluminio de alta pureza $(7 \mathrm{~N})$ fue determinada por la técnica de Espectrofotometría de Absorción Atómica de Llama para detectar las impurezas más frecuentes ( $\mathrm{Fe}, \mathrm{Si}, \mathrm{Mg}, \mathrm{Mn}$ ), el equipo empleado es un Espectrómetro de Emisión Óptica con Plasma Acoplado Inductivamente (ICP-OES), marca Perkin-Elmer, modelo Optima 3000 [18].

Las muestras fueron dimensionadas y cortadas con dimensiones de $10 \times 5 \times 0,5 \mathrm{~mm}^{3}$ y $10 \times 10 \times 1 \mathrm{~mm}^{3}$. A fin de obtener una solución sólida homogénea las probetas de la aleación de aluminio AA6061 fueron sometidas a un tratamiento térmico de solución a $580^{\circ} \mathrm{C}$, durante dos horas, en atmósfera inerte de argón (Ar), e inmediatamente templadas en agua fría. El propósito de este tratamiento térmico es producir una sobresaturación de sitios vacantes, que posteriormente servirán de asientos del proceso de nucleación [18-19].

\subsection{Preparación metalográfica}

Para los ensayos de microscopía las muestras, previo estado de solubilización, fueron sometidas a envejecimientos artificiales mediante recocidos isotérmicos a las temperaturas de: $50,75,85,100,125,150,175,200$, $225,250,275,300,325,350,375,400,425,450,475,500,525,550,575^{\circ} \mathrm{C}$, durante 10 minutos, e inmediatamente se les aplicó un temple en agua fría. La preparación para el análisis metalográfico se realizó por preparación metalográfica convencional, que consiste en el desbaste secuencial, en agua fría, usando papel de lija de carburo de silicio con granulometrías de: 80, 120, 240, 320, 400, 600, 1500 y 2000; seguidamente se practicó un pulido secuencial con polvo de alúmina con granulometrías de: 10, 5, 3, 1 y 0,01 $\mu \mathrm{m}$, hasta lograr superficies especulares.

La observación se realizó en un microscopio óptico (MO) invertido de luz incidente reflejada marca Axiovert 25 CA. Posteriormente se realizaron observaciones y el microanálisis elemental (EDX) en el microscopio electrónico de barrido (MEB), Modelo S-2400 Hitachi, con voltaje de aceleración de $20 \mathrm{kV}$, a una presión entre $10^{-6}$ y $10^{-5}$ torr.

Para los ensayos de microscopía electrónica de transmisión (MET) las muestras fueron lijadas y pulidas por ambos lados (especularmente) hasta espesores entre 0,1 y $0,05 \mathrm{~mm}$, después fueron cortadas en forma de disco de $3 \mathrm{~mm}$ de diámetro, y finalmente el adelgazamiento y agujeramiento por pulido electrolítico en una solución al 20\% de ácido perclórico en metanol en el equipo TENUPOL-3 marca Struers, empleando potenciales entre 10 a $20 \mathrm{~V}$, y corrientes entre 0,1 y $2,0 \mathrm{~A}$, a temperaturas entre -60 y $-40{ }^{\circ} \mathrm{C}$, con tiempos de exposición al electrolito entre 1 y 2 minutos. La observación de las muestras y el registro de imágenes se realizó a temperatura ambiente, se emplearon microscopios PHILIPS CM10 y CM12, con voltajes de aceleración de 100 y $120 \mathrm{kV}$, respectivamente [18-19].

\subsection{Método de medida}

El envejecimiento artificial in situ fue seguido a través de la medida de la resistividad eléctrica mediante la aplicación del método de los cuatro contactos eléctricos. Puesto que la resistividad depende de la estructura atómica y molecular, y de las heterogeneidades presentes y creadas en el material, su medida es un método altamente sensible a las transformaciones experimentadas en estado sólido. Los experimentos se llevaron a cabo a presión atmosférica, en atmósfera de aire, en un horno eléctrico acoplado a un controlador digital de temperatura a través de un termopar tipo $\mathrm{R}$ en contacto con la muestra.

La temperatura de la muestra, la tensión y corriente de alimentación aplicadas $\left(\mathrm{V}_{\mathrm{DC}}=4,5 \mathrm{~V}, \mathrm{I}_{\mathrm{DC}}=500\right.$ $\mathrm{mA})$ y la diferencia de potencial flotante $(\Delta \mathrm{V})$ fueron controladas a través de un sistema electrónico de módulos de acondicionamiento y amplificación de señales analógicas de bajo nivel con una exactitud de $\pm 0,05 \%$ en Span, $\pm 10 \mu \mathrm{V}$. Los módulos están conectados a una tarjeta interfaz de digitalización de señales, con resolución de 50.000 tomas/s, velocidad de conversión analógico-digital de $15 \mu$ s y exactitud de $0,01 \%$.

La interfaz está acoplada al ordenador (PC) para el almacenaje y representación gráfica de los datos. El error experimental estimado es menor al $2 \%$. El primer experimento consistió en medir la resistividad en régimen no-isotérmico a tasas de calentamiento lineal constantes de: $1,5,10,15,20,25,30,40,50$ y 60 
${ }^{\circ} \mathrm{C} / \mathrm{min}$. En el segundo experimento se midió la resistividad en régimen isotérmico a las temperaturas de: 85 , $125,150,175,200,225,250,275$ y $300{ }^{\circ} \mathrm{C}$, durante aproximadamente veinte horas $(20 \mathrm{~h})$ [18-19].

\subsection{Análisis e interpretación de las transformaciones de fase por el modelo de KJMA-MITTEMEIJER}

Ha sido demostrado que las transformaciones de fase producidas por procesos isotérmicos y no-isotérmicos se desarrollan, a menudo de forma simultánea, por mecanismos de reacción conocidos como nucleación, crecimiento e impacto entre partículas.

Esta aseveración es la base del modelo cinético propuesto por Mittemeijer [14-15], y el cual está fundamentado en el hecho de que en las transformaciones activadas térmicamente la historia térmica del espécimen determina su etapa de transformación, y además sugiere que la consideración de reacciones homogéneas e isotérmicas en la teoría de KOLMOGOROV-JOHNSON-MEHL-AVRAMI (KJMA) puede ser extendida a reacciones heterogéneas, isotérmicas y no-isotérmicas mediante la expresión:

$$
\alpha=1-\mathrm{e}^{-\beta^{\mathrm{n}}}
$$

donde $\alpha$ representa la fracción transformada, $\mathrm{n}$ representa el exponente de KJMA; y $\beta$ representa la variable de ruta (número adimensional), y es interpretada como proporcional al número de saltos atómicos. Puesto que la temperatura determina la movilidad atómica, y el tiempo define la duración del proceso, este modelo postula para régimen no-isotérmico:

$$
\beta=\int k(T) d t
$$

donde $\mathrm{k}(\mathrm{T})$ representa la tasa de reacción, que depende del tiempo a través de la temperatura, $\mathrm{T}=\mathrm{T}(\mathrm{t})$. Una ecuación tipo Arrhenius permite representarla de la siguiente forma:

$$
\mathbf{k}(\mathbf{T}) \equiv \mathbf{k}_{\mathbf{o}} \mathbf{e}^{-\frac{E_{\mathbf{a}}}{\mathrm{RT}}} \propto \frac{1}{\tau}
$$

Siendo $\mathrm{k}(\mathrm{T})$ dependiente de la transformación con la temperatura, y es descrita por una energía de activación efectiva global, $\mathrm{E}_{\mathrm{a}}, \mathrm{k}_{\mathrm{o}}$ representa el factor de frecuencia de reacción, $\mathrm{R}$ es la constante universal de los gases $(\mathrm{R}=8,314472 \mathrm{~J} / \mathrm{K}-\mathrm{mol})$. El parámetro $\tau$ representa el tiempo característico de transformación de fase, este tiempo está gobernado por la diferencia de composición precipitado-matriz, por la difusión de especies, por el tamaño de partículas y por el campo de microesfuerzos generados [12, 20].

En el análisis no-isotérmico, con tasa de calentamiento lineal constante, $\varphi=\frac{\mathrm{dT}}{\mathrm{dt}}$, la integral de tiempo en la ecuación 2 es remplazada por una integral de temperatura, y haciendo uso de la ecuación 3 se obtiene una expresión aproximada para $\beta$, dada por:

$$
\beta \cong \frac{T^{2}}{\varphi} \frac{R}{E_{a}} k=\left(\frac{T^{2}}{\varphi}\right)\left(\frac{R_{0}}{E_{a}}\right) \exp \left(-\frac{E_{a}}{R T}\right)
$$

de esta expresión se puede determinar directamente la energía de activación efectiva global mediante la siguiente expresión:

$$
\ln \left(\frac{T_{\alpha}^{2}}{\varphi}\right)=\left(\frac{E_{a}}{R}\right) \frac{1}{T_{\alpha}}+\ln \left(\frac{E_{a}}{R k_{o}} \beta_{\alpha}\right)
$$

al graficar $\ln \left(\frac{\mathrm{T}_{\alpha}^{2}}{\varphi}\right)$ en función de $\frac{1}{\mathrm{~T}_{\alpha}}$, la pendiente de la línea recta es equivalente a la energía de activación, $\mathrm{E}_{\mathrm{a}}$. La determinación de $\mathrm{k}_{\mathrm{o}}$ requiere la adopción de un modelo cinético específico $\beta_{\alpha}$. Sin embargo, se propone para este trabajo un método analítico y gráfico para la determinación de $\beta$ [21].

La fracción transformada, $\alpha$, es obtenida a partir de modelos de difusión [22], siendo dada por: 


$$
\alpha=\frac{\rho_{(t)}-\rho_{\mathbf{o}}}{\rho_{\infty}-\rho_{\mathbf{o}}} \equiv \frac{\operatorname{APP}_{\mathbf{o}}^{t}}{\operatorname{ATP}_{\mathbf{o}}^{\infty}}
$$

, con $0 \leq \alpha \leq 1$. En la ecuación $6, \rho_{(\mathrm{t})}\left(\mathrm{o} \rho_{(\mathrm{T})}\right)$ representa la resistividad eléctrica (u otra propiedad física o mecánica) medida durante el curso de la transformación, $\rho_{\mathrm{o}}$ y $\rho_{\infty}$ corresponden a los valores de resistividad al inicio y al final de la transformación, respectivamente. $\mathbf{A P P}_{\mathbf{0}}^{\mathbf{t}}$ y $\mathbf{A} \mathbf{T} \mathbf{P}_{\mathbf{0}}^{\infty}$ representan el área parcial y total del pico de resistividad, respectivamente.

Una expresión para la tasa de transformación, derivada a partir de las ecuaciones 1 y 2, resulta en:

$$
\frac{\mathrm{d} \alpha}{\mathrm{dt}}=\mathbf{n k}(1-\alpha)\left[\ln (1-\alpha)^{-1}\right]^{\frac{\mathrm{n}-1}{\mathrm{n}}}
$$

En la identificación y caracterización de las transformaciones de fase en el aluminio AA6061, se implementó y aplicó este modelo descrito, considerando las medidas de resistividad eléctrica en regímenes no-isotérmico e isotérmico.

2.5 Análisis e interpretación del comportamiento reológico para tiempos característicos por el modelo de Reiner

La Reología es una ciencia que estudia la deformación y el flujo de la materia, por lo cual tiene como objetivo particular la observación del comportamiento de materiales que experimentan pequeñas deformaciones. En reología la escala del tiempo es reportada en términos de un número adimensional, el Número de Deborah $\left(\mathrm{D}_{\mathrm{e}}\right)$, definido como el cociente entre un tiempo característico del sistema en estudio, tiempo reológico, $(\delta), \mathrm{y}$ el tiempo experimental $\left(t_{\text {exp. }}\right)$ [16], y se expresa como sigue:

$$
D_{\mathrm{e}} \equiv \frac{\text { tiempo característico del sistema en estudio }}{\text { tiempo de observación experimental }}=\frac{\delta}{t_{\text {exp. }}}
$$

de acuerdo a su valor se puede establecer la diferencia entre materiales con tendencia a ser sólidos rígidos y materiales con tendencia a la fluidez. Si el tiempo característico es mayor que el tiempo experimental, el material, para este propósito práctico se comporta como un sólido. Por otra parte, si el tiempo experimental es muy grande, o si el tiempo característico es muy pequeño, en este caso, el material para este propósito práctico se comporta como un fluido. Así, cuanto mayor es $\mathrm{D}_{\mathrm{e}}$, el material es más sólido; cuanto menor es $\mathrm{D}_{\mathrm{e}}$, el material es más fluido. Si $\mathrm{D}_{\mathrm{e}} \geq 1$, el comportamiento es el de un sólido; si $\mathrm{D}_{\mathrm{e}}<<1$, el comportamiento es viscoso; si $\mathrm{D}_{\mathrm{e}} \approx 1$, el comportamiento es viscoelástico. De forma general, todos los materiales son prácticamente viscoelásticos [16, 23].

El estudio del comportamiento reológico del aluminio AA6061 se hizo considerando, en primer lugar, que las medidas de resistividad eléctrica, como una proyección macroscópica de los cambios microestructurales, en régimen isotérmico permiten diferenciar y caracterizar los procesos de deformación y los procesos de relajación de tensiones a nivel de la microestructura del aluminio estudiado; y, en segundo lugar, para categorizar el grado de viscosidad, o viscoelasticidad o solidez de la aleación AA6061, así como también categorizar el nivel de difusión de especies atómicas en el volumen del sólido (alto, medio, bajo) empleando el Número de Deborah establecido por M. Reiner [16].

La interpretación macroestructural ha sido asociada con los tiempos característicos de deformación, de relajación y de estacionariedad, $\lambda$ ( $\lambda \equiv \delta$, ver la ecuación 8 ), en función de la temperatura; la información obtenida ha sido vinculada con el grado de viscosidad, de viscoelasticidad, o de solidez de este material. La interpretación microestructural ha sido asociada con el tiempo característico de transformación de fases, $\tau$ ( $\tau \equiv \delta$, ver la ecuación 8 ), determinado mediante la implementación y aplicación del modelo de KJMAMITTEMEIJER (de la ecuación 7), en función de la temperatura; la información obtenida ha sido vinculada con el nivel de difusión de especies atómicas en el volumen de este material (alto, medio, bajo). 


\section{RESULTADOS Y DISCUSIÓN}

\subsection{Composición química de la aleación estudiada}

La Tabla 1 presenta los valores obtenidos de la composición química de la aleación estudiada, los dados en porcentaje en peso y en porcentaje atómico, y los valores nominales de composición establecidos para el aluminio AA6061 [24], además se reporta la composición determinada para el aluminio comercial de pureza $7 \mathrm{~N}$.

Tabla 1: Composición química de la aleación estudiada, banda de composición química nominal establecida para el alumínio AA6061 [24], y composición química del aluminio 7N. (\% en peso y atómico).

\begin{tabular}{c|c|c|c|c}
\hline ELEMENTO & $\begin{array}{c}\text { AA6061 } \\
\text { (\% en peso) }\end{array}$ & $\begin{array}{c}\text { AA6061 } \\
\text { (\% atómico) }\end{array}$ & $\begin{array}{c}\text { Banda [24] } \\
\text { (\% en peso) }\end{array}$ & $\begin{array}{c}\text { AA1000 - 7N } \\
\text { (\% en peso) }\end{array}$ \\
\hline $\mathrm{Mg}$ & 0,8249 & 0,9178 & $0,8-1,2$ & -- \\
\hline $\mathrm{Si}$ & 0,5560 & 0,5354 & $0,4-0,8$ & ND \\
\hline $\mathrm{Fe}$ & 0,1860 & 0,0901 & 0,7 (máx.) & $0,005 \pm 0,003$ \\
\hline $\mathrm{Cu}$ & 0,1750 & 0,0745 & $0,15-0,4$ & ND \\
\hline $\mathrm{Cr}$ & 0,0686 & 0,0357 & $0,04-0,35$ & $\mathrm{ND}$ \\
\hline $\mathrm{Mn}$ & 0,0121 & 0,0060 & 0,15 (máx.) & -- \\
\hline $\mathrm{Ti}$ & 0,0062 & 0,0035 & 0,15 (máx.) & ND \\
\hline $\mathrm{Zn}$ & 0,0028 & 0,0012 & 0,25 (máx.) & ND \\
\hline otros & 0,0699 & 0,0110 & - & ND \\
\hline Balance de Al & 98,0985 & 98,3248 & 98 & \\
\hline
\end{tabular}

ND: No Detectado.

El análisis elemental confirmó que todos los elementos reportados en la Tabla 1 se encuentran dentro de los intervalos establecidos para el aluminio AA6061, y los aleantes principales de la aleación estudiada son el $\mathrm{Mg}$ y el $\mathrm{Si}$; se determinó la razón $\mathrm{Mg}$ : $\mathrm{Si}$, en \% atómico, resultando una relación de $\mathrm{Mg} / \mathrm{Si}=1,71$. Este valor indica un ligero exceso de $\mathrm{Si}$ respecto al contenido de $\mathrm{Mg}$, ya que su relación debe ser de 2:1 correspondiente a la estequiometría del compuesto $\mathrm{Mg}_{2} \mathrm{Si}$, fase secundaria principal para este sistema, que establece una razón de $\mathrm{Mg} / \mathrm{Si}=1,73$. Además este exceso de $\mathrm{Si}$ se combina, particularmente, con el Fe y el abundante Al, para formar fases constitutivas durante el proceso de colada. Esto último ha sido confirmado a través del análisis de la microestructura, a continuación.

\subsection{Análisis de la microestructura}

La Figura 1 muestra imágenes de MET correspondientes a la aleación de aluminio AA6061: (a) en estado homogeneizado; y (b, c, d) envejecidas artificialmente a: 85,300 y $425{ }^{\circ} \mathrm{C}$, respectivamente.
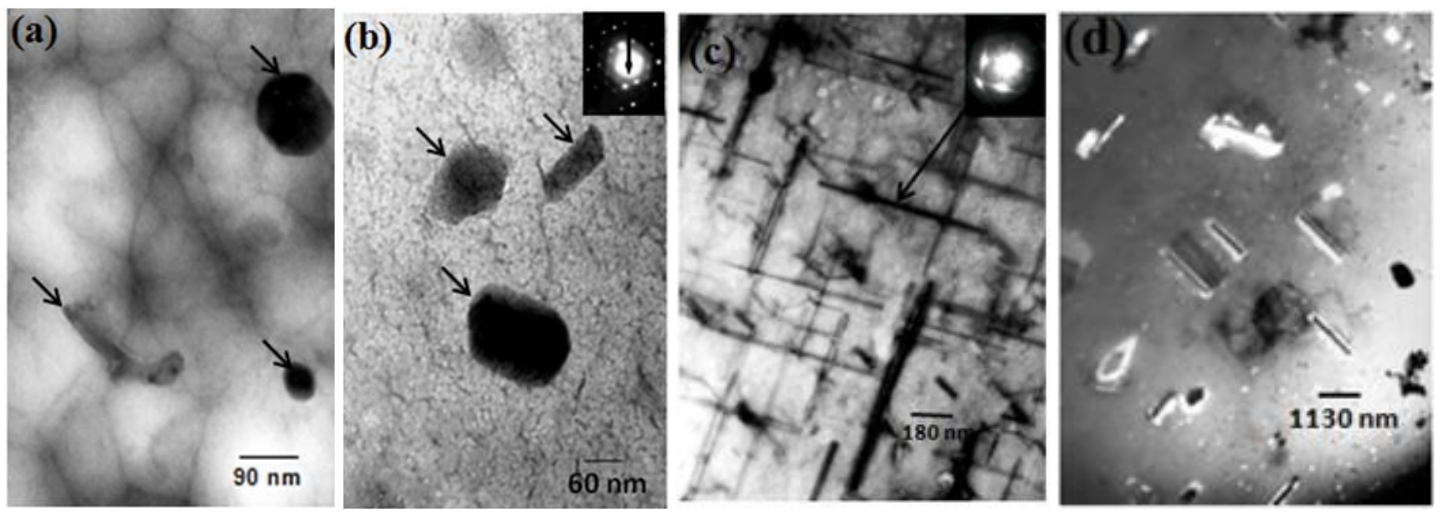
Figura 1: Imágenes de MET de la aleación AA6061: (a) estado homogeneizado; (b, c y d) envejecidas artificialmente a 85,300 y $425{ }^{\circ} \mathrm{C}$, respectivamente. En (a) y (b) los nano-precipitados señalados con flechas corresponden a fases constitutivas a base de $\mathrm{Fe}$ o $\mathrm{Cu}$ identificadas por EDX (ver Figuras 2 y 3, y Tablas 2 y 3). El patrón de difracción de electrones en la parte superior derecha en (b) corresponde a la fase $\mathrm{Al}_{2} \mathrm{Cu}$. En (c) se muestra una matriz con gran cantidad de precipitados aciculares con tamaños mayores a $800 \mathrm{~nm}$ y corresponden a embriones de la fase $\beta$ y a las fases metaestables $\beta$ “ y $\beta^{\prime}$. El patrón de difracción de electrones en la parte superior derecha de la imagen corresponde al precipitado acicular, señalado por la flecha, con composición correspondiente a Mg-Si determinada por EDX. En (d) se muestran precipitados de forma cilíndrica con tamaños mayores a $1000 \mathrm{~nm}$ y composición correspondiente a $\mathrm{Mg}-\mathrm{Si}$ determinada por EDX, estos precipitados corresponden a la fase estable $\beta\left(\mathrm{Mg}_{2} \mathrm{Si}\right)$.

La imagen de la Figura 1a, aleación AA6061 en estado homogeneizado, muestra los límites de grano en la matriz de aluminio, además de contener nano-precipitados intermetálicos correspondientes a las fases constitutivas de $\mathrm{Fe}-\mathrm{Si}, \mathrm{Al}-\mathrm{Cu}$, entre otras (patrones de difracción de electrones no presentados). La imagen de la Figura 1b, aleación AA6061 envejecida a $85{ }^{\circ} \mathrm{C}$, muestra la matriz de aluminio cargada con diminutos nano-precipitados en formación en correspondencia con el abundante $\alpha_{\mathrm{Al}}+(\mathrm{Mg}-\mathrm{Si})$ reportado por espectroscopía EDX, la morfología de estos diminutos nano-precipitados no ha sido definida, y su distribución es muy densa.

Además, se observan, en la Figura 1b, conglomerados de dislocaciones. También, en la Figura 1b, se observan nano-precipitados intermetálicos. El patrón de difracción de electrones, parte superior derecha de la Figura $1 \mathrm{~b}$, corresponde a la fase $\mathrm{Al}_{2} \mathrm{Cu}$. Los nano-precipitados señalados con flechas cortas, en las Figuras (1a y 1b), fueron caracterizados e identificados por espectroscopía EDX, y corresponden a fases intermetálicas a base de $\mathrm{Fe}$ o de $\mathrm{Cu}$, tales como: $\mathrm{Fe}-\mathrm{Si}, \mathrm{Al}-\mathrm{Cu}$, Al-Fe, Al-Fe-Si, Al-Mg-Si-Cu, Al-Mg-Si-Fe, entre otras, y en correspondencia con los porcentajes de composición de la aleación; estos nano-precipitados presentan altos puntos de fusión como resultante del proceso de colada. La Figura 2 muestra una imagen de MEB y espectros EDX para la aleación AA6061 en estado homogeneizado, y la Tabla 2 presenta los resultados cualitativos y cuantitativos dados por los espectros para los puntos analizados; también, en la Figura 3 se muestra una imagen de MEB y espectros EDX para la aleación AA6061 envejecida a $85^{\circ} \mathrm{C}$, y la Tabla 3 presenta los resultados cualitativos y cuantitativos dados por los espectros para los puntos analizados. La imagen de la Figura $1 \mathrm{c}$, envejecida a $300{ }^{\circ} \mathrm{C}$, muestra una matriz de aluminio con gran cantidad de precipitados aciculares con tamaños mayores a $800 \mathrm{~nm}$, y corresponden a embriones de la fase $\beta$ y a las fases metaestables $\beta^{\prime \prime}$ y $\beta^{\prime}$.

El patrón de difracción de electrones en la parte superior derecha de la imagen corresponde al precipitado acicular, señalado por la flecha, con composición correspondiente a Mg-Si determinada por EDX (no presentada). Estos precipitados actúan como centros captores y/o dispersores de los electrones de conducción elevando la resistividad eléctrica. Junto con el anclaje de las dislocaciones contribuyen al endurecimiento de la aleación. La imagen en la Figura 1d, aleación envejecida a $425^{\circ} \mathrm{C}$, muestra precipitados de forma cilíndrica con tamaños mayores a $1000 \mathrm{~nm}$ y con composición correspondiente a Mg-Si determinada por EDX (no presentada), estos precipitados corresponden a la fase estable $\beta\left(\mathrm{Mg}_{2} \mathrm{Si}\right)$, también identificados por otros autores $[18,21]$. 


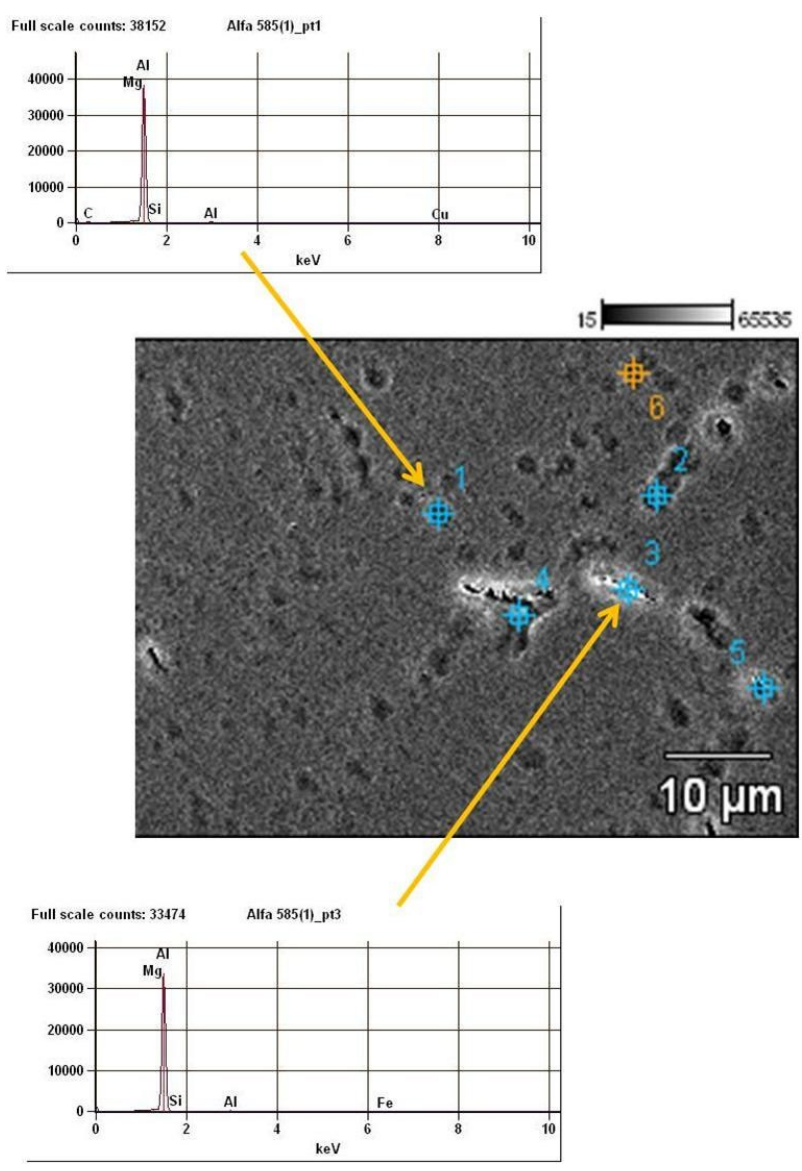

Figura 2: Imagen de MEB de electrones secundarios (magnificación x2000) de la aleación de aluminio AA6061 en estado homogeneizado. También se muestran espectros EDX del análisis puntual para algunas de las regiones seleccionadas (puntos 1 y 3 ).

Tabla 2:Aleación de alumínio AA6061 en estado homogeneizado. Composición correspondiente al análisis puntual representado por los espectros EDX (línea K excitada), porcentaje en peso (\%) y porcentaje de error $( \pm \sigma)$. Puntos seleccionados en la muestra de la aleación AA6061 mostrada en la Figura 2.

\begin{tabular}{|c|c|c|c|c|c|}
\hline $\begin{array}{c}\text { REGIÓN DE LA } \\
\text { MUESTRA }\end{array}$ & Al-K & Mg-K & Si-K & Fe-K & Cu-K \\
\hline$\alpha_{S S S} 580 \_p u n t o 1$ & $97,96 \pm 0,38$ & $1,84 \pm 0,03$ & $0,18 \pm 0,02$ & & $0,02 \pm 0,06$ \\
\hline$\alpha_{\text {SSS }} 580 \_p$ punto2 & $97,55 \pm 0,38$ & $1,77 \pm 0,02$ & $0,49 \pm 0,02$ & & \\
\hline$\alpha_{S S S} 580 \_$punto3 & $97,30 \pm 0,39$ & $1,72 \pm 0,03$ & $0,07 \pm 0,02$ & $0,91 \pm 0,05$ & \\
\hline$\alpha_{S S S} 580 \_p u n t o 4$ & $98,07 \pm 0,39$ & $1,79 \pm 0,03$ & $0,14 \pm 0,02$ & & \\
\hline$\alpha_{\text {SSS }} 580 \_p u n t o 5$ & $97,70 \pm 0,38$ & $1,82 \pm 0,02$ & $0,48 \pm 0,03$ & & \\
\hline$\alpha_{S S S} 580 \_$punto6 & $97,57 \pm 0,38$ & $1,87 \pm 0,03$ & $0,56 \pm 0,03$ & & \\
\hline
\end{tabular}




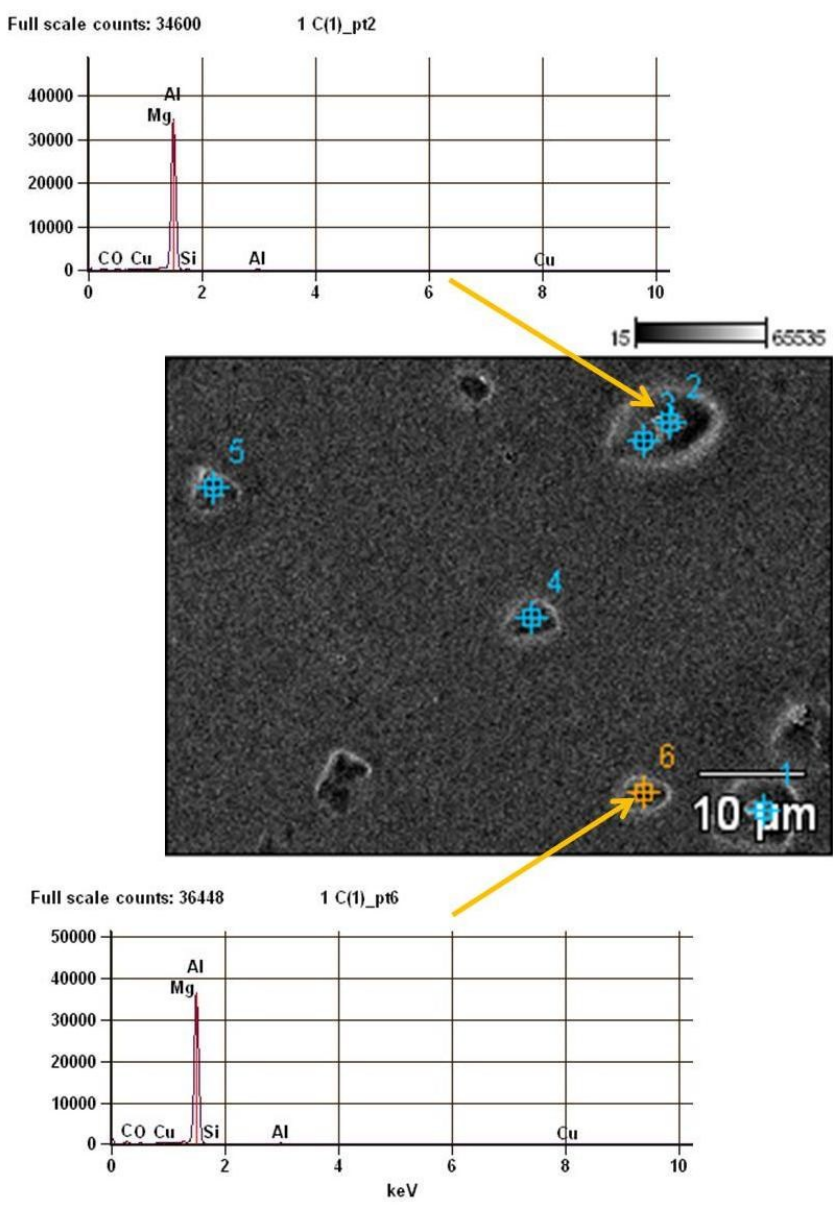

Figura 3: Imagen de MEB de electrones secundarios (magnificación x2000) de la aleación de aluminio AA6061 envejecida artificialmente a $85^{\circ} \mathrm{C}$ durante $10 \mathrm{~min}$. También se muestran espectros EDX del análisis puntual para algunas de las regiones seleccionadas (puntos 2 y 6 ).

Tabla 3: Aleación de aluminio AA6061 en estado envejecido a $85^{\circ} \mathrm{C}$. Composición correspondiente al análisis puntual representado por los espectros EDX (línea K excitada), porcentaje en peso (\%) y porcentaje de error $( \pm \sigma)$. Puntos seleccionados en la muestra de la aleación AA6061 mostrada en la Figura 3.

\begin{tabular}{c|c|c|c|c|c}
\hline $\begin{array}{c}\text { REGIÓN DE LA } \\
\text { MUESTRA }\end{array}$ & Al-K & Mg-K & Si-K & Fe-K & Cu-K \\
\hline $\boldsymbol{M ( 8 5 ^ { \circ } \boldsymbol { C } ) \text { punto1 }}$ & $97,49 \pm 0,39$ & $1,85 \pm 0,03$ & $0,66 \pm 0,03$ & & \\
\hline $\boldsymbol{M ( 8 5 ^ { \circ } \boldsymbol { C } ) \text { punto2 }}$ & $97,29 \pm 0,38$ & $1,87 \pm 0,03$ & $0,79 \pm 0,03$ & & $0,06 \pm 0,07$ \\
\hline $\boldsymbol{M}\left(\mathbf{8 5}^{\circ} \boldsymbol{C}\right)$ punto3 & $97,63 \pm 0,38$ & $1,84 \pm 0,03$ & $0,52 \pm 0,03$ & & \\
\hline $\boldsymbol{M ( 8 5 ^ { \circ } \mathbf { C } ) \text { punto4 }}$ & $97,98 \pm 0,38$ & $1,76 \pm 0,03$ & $0,26 \pm 0,02$ & & \\
\hline $\boldsymbol{M ( 8 5 ^ { \circ } \boldsymbol { C } ) \text { punto5 }}$ & $98,05 \pm 0,38$ & $1,72 \pm 0,02$ & $0,24 \pm 0,02$ & & \\
\hline $\boldsymbol{M ( 8 5 ^ { \circ } \mathbf { C } ) \text { punto6 }}$ & $97,66 \pm 0,38$ & $1,75 \pm 0,02$ & $0,28 \pm 0,02$ & & $0,32 \pm 0,06$ \\
\hline
\end{tabular}

\subsection{Resistividad eléctrica}

\subsubsection{Resistividad eléctrica no-isotérmica: efecto de los elementos aleantes e impurezas}

La Figura 4a muestra el gráfico de la resistividad eléctrica, obtenido en régimen no-isotérmico, en función de la temperatura dinámica, e identificado como: curva (A) aluminio comercial de alta pureza 7N (99,99999 \%); curva (B) aluminio de pureza 99,995\% (datos tomados de la referencia [17]); y curva (C) aleación estudiada AA6061 en estado homogeneizado. Las curvas (A) y (B) presentan comportamientos lineales, mientras que la curva (C) muestra un comportamiento no-lineal; estas curvas fueron generadas a una tasa de calentamiento 
de $1{ }^{\circ} \mathrm{C} / \mathrm{min}$., desde la temperatura ambiente hasta aproximadamente $600{ }^{\circ} \mathrm{C}$. La Figura $4 \mathrm{~b}$ muestra el gráfico de la resistividad eléctrica normalizada correspondiente a la curva (C), dada en la Figura 4a. La normalización de la curva (C) se efectuó considerando la curva linealizada de la resistividad del aluminio de pureza $7 \mathrm{~N}$ como referencial, con base en OCHOA-M. y colaboradores [25]. El gráfico con la curva normalizada mejora la percepción visual de los cambios experimentados por la resistividad, y contribuye a flexibilizar el proceso iterativo de cálculos durante la ejecución de algoritmos de optimización. Los gráficos para otras tasas de calentamiento (no presentados) muestran comportamientos similares en comparación con la curva (C) de la Figura 4a, éstos han sido reportados por OCHOA-M. [21].
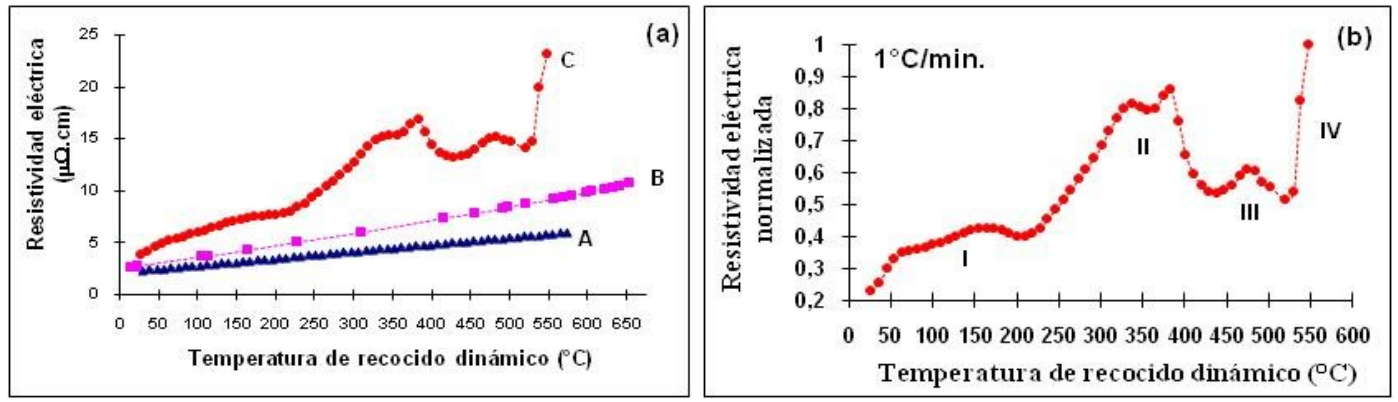

Figura 4a: Gráficos de la Resistividad eléctrica no-isotérmica en función de la temperatura dinámica mostrando la curva (A) del aluminio comercial de pureza 7N (99,99999\%), la curva (B) de un aluminio de pureza 99,995\% (datos tomados de la referencia [17]), y la curva (C) de la aleación AA6061 en estado homogeneizado. Figura 4b. Gráfico de la Resistividad eléctrica normalizada, con base al máximo valor de la curva (C) y considerando la curva linealizada (A) como referencial en la Figura 4a, se indican las regiones de transformaciones de fase (I, II, III e IV).

A partir de la Figura 4a se observa que las curvas de resistividad (A) y (B) presentan un comportamiento lineal, y obedecen a una ecuación de la forma: $\rho=\rho_{o}+\alpha \Delta T$. Entretanto, la curva de resistividad (C) muestra un comportamiento no-lineal, presentando fluctuaciones con picos de diferentes anchos y alturas, estas fluctuaciones de la resistividad están asociadas directamente a la presencia de los elementos aleantes e impurezas, además de las fases secundarias formadas $\mathrm{y}$ en transformación, y contribuyen significativamente en el rompimiento del comportamiento lineal de esta propiedad física.

A la temperatura ambiente la resistividad eléctrica presenta, para las curvas (A), (B) y (C) (ver Figura

4a), los valores: $\rho_{\mathbf{o}}^{\mathrm{A}}=\mathbf{2 , 3 2} \boldsymbol{\mu} \boldsymbol{\Omega} . \mathbf{c m}, \rho_{\mathbf{o}}^{\mathrm{B}}=\mathbf{2 , 6 9} \boldsymbol{\mu} \boldsymbol{\Omega} . \mathbf{c m}$ y $\boldsymbol{\rho}_{\mathbf{o}}^{\mathrm{C}}=\mathbf{3 , 8 8} \boldsymbol{\mu} \boldsymbol{\Omega} . \mathbf{c m}$, respectivamente. Y los valores máximos, a altas temperaturas, son: $\rho_{\text {máx. }}^{A}=\mathbf{5 , 9 7} \boldsymbol{\mu} \boldsymbol{\Omega} \mathbf{c m}\left(\approx 575^{\circ} \mathrm{C}\right), \boldsymbol{\rho}_{\text {máx. }}^{\mathrm{B}}=\mathbf{9 , 3 5} \boldsymbol{\mu} \boldsymbol{\Omega} . \mathbf{c m}(\approx 570$ $\left.{ }^{\circ} \mathrm{C}\right)$ y $\rho_{\text {máx. }}^{\mathrm{C}}=23,09 \mu \Omega \cdot \mathbf{c m}\left(\approx 550^{\circ} \mathrm{C}\right)$, respectivamente.

La marcada diferencia entre los valores de resistividad aporta una valiosa información relacionada con la influencia que ejercen los elementos aleantes, impurezas, las fases secundarias formadas y las fases constitutivas presentes en estos aluminios, esta influencia es reflejada macroscópicamente a través de la resistividad eléctrica. Por otro lado, la trayectoria seguida por la curva de resistividad de la aleación AA6061 (curva C) permitió la observación y demarcación de cuatro grandes regiones de transformaciones de fase bien definidas e indicadas por las regiones I, II, III y IV (ver la Figura 4b).

La implementación del modelo de KJMA-MITTEMEIJER, aplicado a procesos no-isotérmicos asociados a estas transformaciones, permitió la determinación de los parámetros cinéticos con el uso de la ecuación 5. Así, se obtiene que en la región I donde se presenta una pequeña meseta comprendida entre la temperatura am-biente hasta aproximadamente $190^{\circ} \mathrm{C}$, con un máximo a $150{ }^{\circ} \mathrm{C}$; y con energías de activación, $\left(\mathrm{E}_{\mathrm{a}}^{\mathrm{I}}\right)$, comprendidas entre 20 y $60 \mathrm{~kJ} / \mathrm{mol}(0,22$ y $0,66 \mathrm{eV} / \mathrm{at})$, estos valores son coherentes con los resultados de otros autores [26-32], con el empleo de técnicas similares, que resultaron con valores entre 20 y $70 \mathrm{~kJ} / \mathrm{mol}$ $(0,22$ y $0,77 \mathrm{eV} / \mathrm{at})$. Asociando e interpretando estas energías con procesos cinéticos variados desde el tránsito de una reacción rápida a una reacción lenta, están relacionadas con las energías enlazantes de formación de mono y di-vacancias, con las energías de activación para la difusión, con la energía libre enlazante entre un átomo de Si y una vacancia, y con las energías de migración de átomos de soluto ( $\mathrm{Mg}, \mathrm{Si})$. Relacionando las imágenes de MET de las Figuras (1a y 1b) con el origen de esta meseta (región I) se puede considerar como un efecto de la formación y conformación de agregados de soluto y embriones de fases aciculares metaestables. Además, la conformación de los agregados de soluto se debe al efecto de la tensión eléctrica aplicada y 
al aumento paulatino de la temperatura que acelera el proceso de nucleación.

En la región II se muestra un pico alto y ancho entre 220 y $420{ }^{\circ} \mathrm{C}$, con un máximo a $350{ }^{\circ} \mathrm{C}$; las energías de activación, ( $\left.\mathbf{E}_{\mathbf{a}}^{\mathrm{II}}\right)$, están entre 90 y $120 \mathrm{~kJ} / \mathrm{mol}(0,99$ y $1,32 \mathrm{eV} / \mathrm{at})$, estos valores están dentro del intervalo de valores de energías de activación para la difusión de $\mathrm{Mg}$ y $\mathrm{Si}$ en Al, entre 80 y $150 \mathrm{~kJ} / \mathrm{mol}(0,88$ y 1,64 eV/at), determinados por otros autores [27-28]. Según KÊ T. S., [33], un ancho y alto pico, asociado con la fricción interna, se situa a la temperatura de $300^{\circ} \mathrm{C}$ para un aluminio policristalino de alta pureza. La imagen de MET de la Figura 1c muestra precipitados aciculares con tamaños mayores a $800 \mathrm{~nm}$, y están relacionados con la región II, la anchura y altura del pico ubicado a $350{ }^{\circ} \mathrm{C}$ son proporcionales a la concentración, tamaño y transformación de estas fases. La región III localizada entre 420 y $520{ }^{\circ} \mathrm{C}$, presenta un pico a $475^{\circ} \mathrm{C}$, y sus energías de activación, $\left(\mathbf{E}_{\mathbf{a}}^{\text {III }}\right)$, están entre 400 y $470 \mathrm{~kJ} / \mathrm{mol}(4,40$ y $5,17 \mathrm{eV} / \mathrm{at})$. La imagen de MET en la Figura 1d muestra precipitados cilíndricos con tamaños mayores a $1000 \mathrm{~nm}$, y están relacionados con la región III por lo cual dan origen a este pequeño pico, y se vincula con la formación y consolidación de la fase estable $\beta$. En la región IV, localizada a temperaturas mayores a $525{ }^{\circ} \mathrm{C}$, se inicia el proceso de redisolución de las fases precipitadas.

\subsubsection{Resistividad eléctrica isotérmica: procesos de deformación y relajación}

La Figura 5a muestra el gráfico con las curvas de la resistividad eléctrica, obtenido en régimen isotérmico, en función del tiempo de recocido estático in situ de la aleación AA6061 en estado homogeneizado, para las temperaturas de $85,120,150$ y $175^{\circ} \mathrm{C}$ (indicadas). Estos datos (Figura 5a) son normalizados y resultan en el gráfico de la Figura $5 b$, donde se muestran las curvas de la resistividad eléctrica normalizada a su valor máximo, para las mismas temperaturas de recocido. Otras curvas fueron obtenidas a otras temperaturas de recocido in situ y presentan comportamientos similares y no son presentadas para simplificar y evidenciar las temperaturas más importantes que fueron seleccionadas.
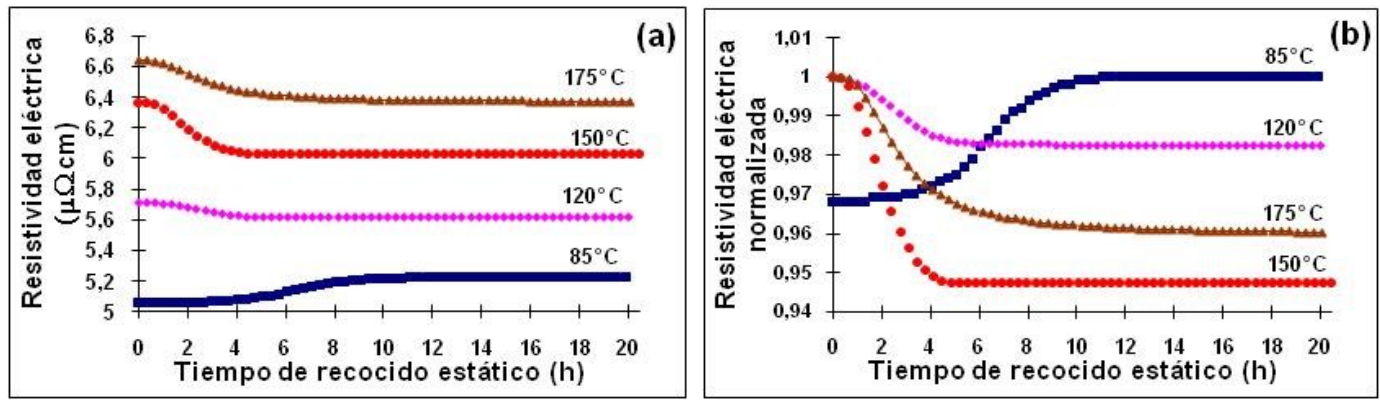

Figura 5: Aleación de alumínio AA6061 en estado homogeneizado. (a) Resistividad eléctrica isotérmica en función del tiempo de recocido estático in situ (temperaturas indicadas). (b) Resistividad eléctrica normalizada, correspondiente a la Figura 5a.

Comparando las curvas de la Figura $5 \mathrm{a}$ y la curva $\mathrm{C}$ de la Figura 4a, se encuentra que existe una gran concordancia entre los valores de resistividad obtenidos para ambas medidas. Por otro lado, en la curva de la Figura $5 \mathrm{~b}$, se observa que la resistividad a $85^{\circ} \mathrm{C}$ inicialmente aumenta y luego permanece estacionaria, este comportamiento está asociado a un proceso de deformación permanente. A las temperaturas de $120,150 \mathrm{y}$ $175^{\circ} \mathrm{C}$ la resistividad muestra un decremento paulatino hasta permanecer estacionaria, en este caso, el comportamiento está asociado a un proceso de relajación de tensiones. Con relación a las curvas de resistividad, en la Figura 5b, su comportamiento en relajación presenta una fuerte correlación con las curvas de resistencia eléctrica normalizada para la aleación $\mathrm{Al}-12.5 \% \mathrm{Si}-1 \% \mathrm{Mg}$ obtenidas por Tawfik et al. [27].

\subsubsection{Tasa de la resistividad eléctrica}

A fin de evidenciar y obtener información acerca de la dinámica de los procesos de deformación y de relajación se determinó la tasa de la resistividad mediante el cálculo de las derivadas en función del tiempo a las curvas de la resistividad eléctrica mostradas en la Figura 5a (o Figura 5b). La curva de resistividad eléctrica a $85^{\circ} \mathrm{C}$ (Figura 5a o Figura $5 \mathrm{~b}$ ) es derivada, y resulta en el gráfico de la Figura $6 \mathrm{a}, \frac{\mathrm{d} \rho}{\mathrm{dt}}$. A partir del gráfico de la

Figura 6a se observa que inicialmente la tasa (en términos de valor absoluto) experimenta un acelera- 
miento hasta un valor máximo, aproximadamente a las seis horas $(6 \mathrm{~h})$ de recocido in situ, e inmediatamente después la tasa experimenta un desaceleramiento hasta alcanzar un estado estacionario, aproximadamente a las doce horas $(12 \mathrm{~h})$ de recocido in situ, la estacionariedad permanece hasta que finaliza el experimento, es decir se alcanza un estado de deformación permanente a esta temperatura. Por otro lado, y similarmente, las curvas de resistividad eléctrica a 120,150 y $175^{\circ} \mathrm{C}$ (Figura 5 a o Figura $5 \mathrm{~b}$ ) son derivadas y resulta el gráfico de la Figura $6 \mathrm{~b}, \frac{\mathrm{d} \rho}{\mathrm{dt}}$, con las temperaturas indicadas para cada curva. A partir del gráfico de la Figura $6 \mathrm{~b}$ se observa que inicialmente las tasas (en términos de valor absoluto), para cada curva, experimentan un aceleramiento hasta un valor máximo, alrededor de las dos horas $(2 \mathrm{~h})$, e inmediatamente después las tasas experimentan un desaceleramiento hasta alcanzar un estado estacionario, alrededor de las cuatro horas $(4 \mathrm{~h})$, la estacionariedad va a permanecer hasta que finaliza el experimento, es decir se alcanza un estado de relajación de tensiones para cada una de las temperaturas consideradas. Finalmente, los gráficos de las Figuras (6a y 6b) permiten observar y confirmar que los procesos de deformación y de relajación, vinculados directamente con el proceso de transformación de fases, y asociados a las vibraciones de la red [34], se desarrollan en varias etapas y con tasas variando en el tiempo (no constantes), así, las curvas muestran tres etapas de: aceleramiento, desaceleramiento y estacionariedad. Además, se observa en estos gráficos (Figuras 6a y 6b) que el proceso de deformación es más lento que los procesos de relajación a las temperaturas consideradas.

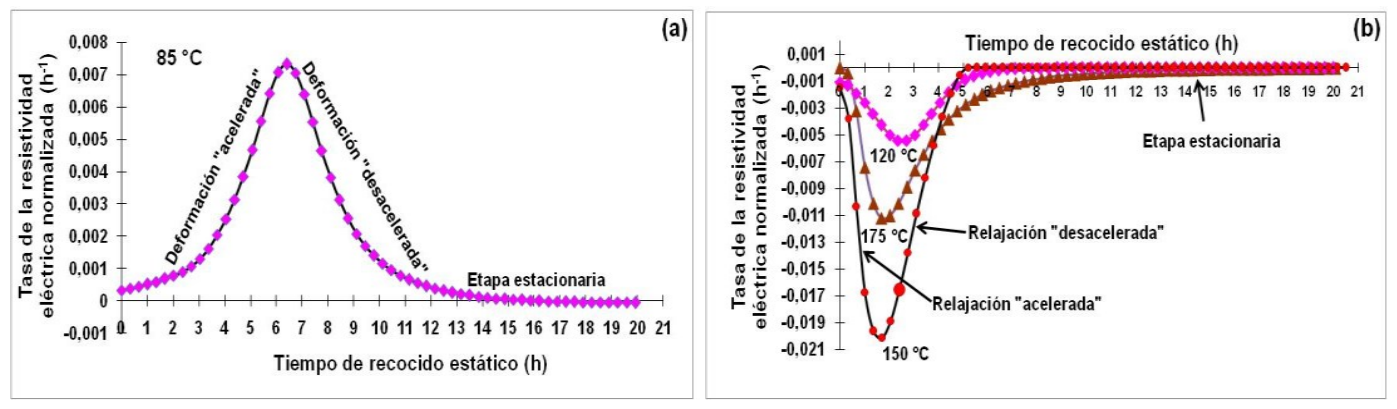

Figura 6: Tasa de la resistividad eléctrica, $\frac{\mathrm{d} \rho}{\mathrm{dt}}$, en función del tiempo de recocido in situ, a las temperaturas indicadas. (a) Proceso de deformación $\left(85^{\circ} \mathrm{C}\right)$. (b) Proceso de relajación de tensiones $\left(120,150\right.$ y $\left.175^{\circ} \mathrm{C}\right)$. En todas las curvas se observan tres etapas: aceleramiento, desaceleramiento y estacionariedad. Las líneas continuas que unen los puntos experimentales representan ajustes polinómicos.

\subsection{Número de Deborah}

\subsubsection{Tiempo característico de deformación y relajación}

Las Figuras (7a y 7b) muestran los gráficos que representan el comportamiento del Número de Deborah $\left(\mathrm{D}_{\mathrm{e}}\right)$ obtenido a partir de la ecuación 8 empleando los tiempos característicos para los procesos de deformación, relajación y estacionariedad, $\lambda(\lambda \equiv \delta$, ver ecuación 8$)$, en función de la temperatura de recocido estático (además se consideran puntos experimentales para otras temperaturas no presentadas por razones de simplificación). El gráfico de la Figura 7a muestra las curvas correspondientes a las etapas de aceleramiento ( $1^{\text {ra }}$ etapa), desaceleramiento ( $2^{\text {da }}$ etapa), y la etapa combinada (suma aritmética de las curvas de aceleramiento y desaceleramiento, equivalente a la finalización de la transformación), estas etapas están indicadas en los gráficos (a) y (b) de la Figura 6. El gráfico de la Figura 7b muestra la curva correspondiente a la etapa estacionaria ( $3^{\text {ra }}$ etapa), esta etapa está indicada en los gráficos (a) y (b) de la Figura 6. En los gráficos mostrados en las Figuras (7a y 7b) las líneas continuas y la línea a trazos, que unen los puntos experimentales, representan ajustes polinómicos que indican las tendencias seguidas. 

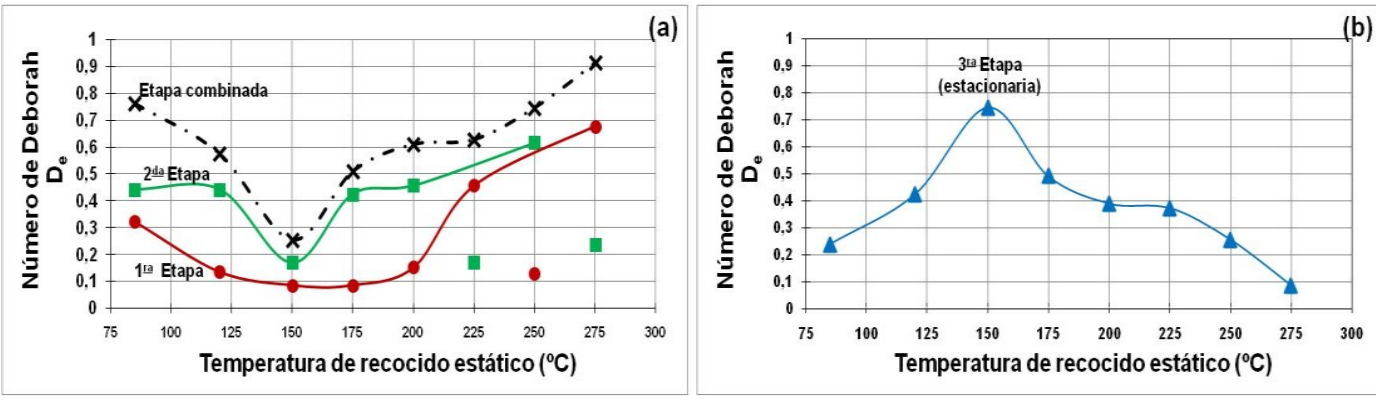

Figura 7: Número de Deborah $\left(D_{e}\right)$ determinado para los procesos de deformación, relajación y estacionariedad, en función de la temperatura de recocido estático. (a) Etapas de aceleramiento, desaceleramiento, y combinada (aceleramiento + desaceleramiento). (b) Etapa estacionaria. Las líneas continuas y la línea a trazos, que unen los puntos experimentales, representan ajustes polinómicos que muestran las tendencias seguidas.

Las etapas de aceleración y desaceleración, en la tasa de la resistividad, están vinculadas directamente con la transformación de fases en particular, y la suma aritmética de estas dos etapas, o etapa combinada, pone punto final a la transformación considerada, dada a una temperatura particular.

Considerando el gráfico de la Figura 7a, se observa que, en primer lugar, las tres curvas (aceleramiento y desaceleramiento, y combinada) muestran un comportamiento paralelo entre sí. En segundo lugar, las tres curvas, a las temperaturas de 85,120 y $150{ }^{\circ} \mathrm{C}$, experimentan un decrecimiento un tanto exponencial, es decir, el Número de Deborah decrece entorno al intervalo $0,085 \leq \mathrm{D}_{\mathrm{e}} \leq 0,760$, este comportamiento se interpreta macroscópicamente como un ablandamiento experimentado por la aleación de aluminio AA6061, de acuerdo a este intervalo de valores se pasa de un estado viscoelástico a un estado viscoso. En tercer lugar, las tres curvas, a partir de los $150{ }^{\circ} \mathrm{C}$ hasta $275^{\circ} \mathrm{C}$, experimentan un crecimiento un tanto exponencial, el Número de Deborah crece entorno al intervalo $0,085 \leq \mathrm{D}_{\mathrm{e}} \leq 0,920$, este comportamiento se interpreta macroscópicamente como un endurecimiento experimentado por la aleación de aluminio AA6061, el caso contrario al anterior, se pasa de un estado viscoso a un estado viscoelástico. Finalmente, las tres curvas registran un mínimo de ablandamiento alrededor de la temperatura de $150{ }^{\circ} \mathrm{C}$. La curva de aceleramiento presenta los valores menores de $\mathrm{D}_{\mathrm{e}}$; la curva de desaceleramiento presenta los valores intermedios de $\mathrm{D}_{\mathrm{e}}$; y al finalizar el proceso de transformación, representado por la curva combinada, se obtienen los valores mayores de $\mathrm{D}_{\mathrm{e}}$, la aleación de aluminio AA6061, finalizado el proceso de transformación, se endurece.

Considerando el gráfico de la Figura $7 \mathrm{~b}$, se observa que la curva que representa la etapa estacionaria, a las temperaturas de 85,120 y $150^{\circ} \mathrm{C}$, experimenta un crecimiento un tanto exponencial, el Número de Deborah crece entorno al intervalo $0,24 \leq \mathrm{De} \leq 0,75$, este comportamiento se interpreta macroscópicamente como un endurecimiento experimentado por la aleación de aluminio AA6061, de acuerdo a este intervalo de valores de De, se pasa de un estado viscoso a un estado viscoelástico. Por otro lado, la curva, a partir de los 150 ${ }^{\circ} \mathrm{C}$ hasta $275^{\circ} \mathrm{C}$, experimenta un decrecimiento un tanto exponencial, el Número de Deborah decrece entorno al intervalo $0,085 \leq \mathrm{De} \leq 0,75$, este comportamiento se interpreta macroscópicamente como un ablandamiento experimentado por la aleación de aluminio AA6061, el caso contrario al anterior, se pasa de un estado viscoelástico a un estado viscoso. Este ablandamiento es producto del largo periodo de calentamiento (texp. = 20,5 h), y al aumento de la temperatura, a que ha sido sometida la aleación AA6061, de allí el decrecimiento continuo experimentado por el valor de De.

Finalmente, las curvas que describen el comportamiento del Número de Deborah (De) muestran un punto de inflexión alrededor de $150{ }^{\circ} \mathrm{C}$. Para esta temperatura se observa que durante la transformación se tiene $0,085 \leq \mathrm{De} \leq 0,260$ (ablandamiento), mientras que después de la transformación De $=0,75$ (endurecimiento). Esta situación de tránsito de un estado de ablandamiento durante la transformación (estado viscoso) a un estado de endurecimiento después de la transformación (estado viscoelástico) invita a considerar la temperatura de $150^{\circ} \mathrm{C}$ como un referencial tecnológico para procesos de endurecimiento de estas aleaciones de aluminio. 


\subsubsection{Tiempo característico de transformación de fases}

\subsubsection{Fracción transformada y tasa de transformación}

La Figura 8a muestra el gráfico de la fracción transformada, $\alpha$, obtenida con base a los resultados reportados en la Figura 5 y la ecuación 6, en función del tiempo de recocido, a las temperaturas de 85, 120, 175, y $150{ }^{\circ} \mathrm{C}$ (indicadas). Cada curva de la fracción transformada, $\alpha$, de la Figura 8a, es derivada respecto al tiempo, y resulta en el gráfico de la Figura $8 \mathrm{~b}$, que representa el gráfico de la tasa de transformación, $\frac{\mathrm{d} \alpha}{\mathrm{dt}}$, en función del tiempo de recocido, a las mismas temperaturas indicadas. En ambos gráficos (Figuras 8a y 8b) las líneas continuas que unen los puntos experimentales representan ajustes polinómicos.
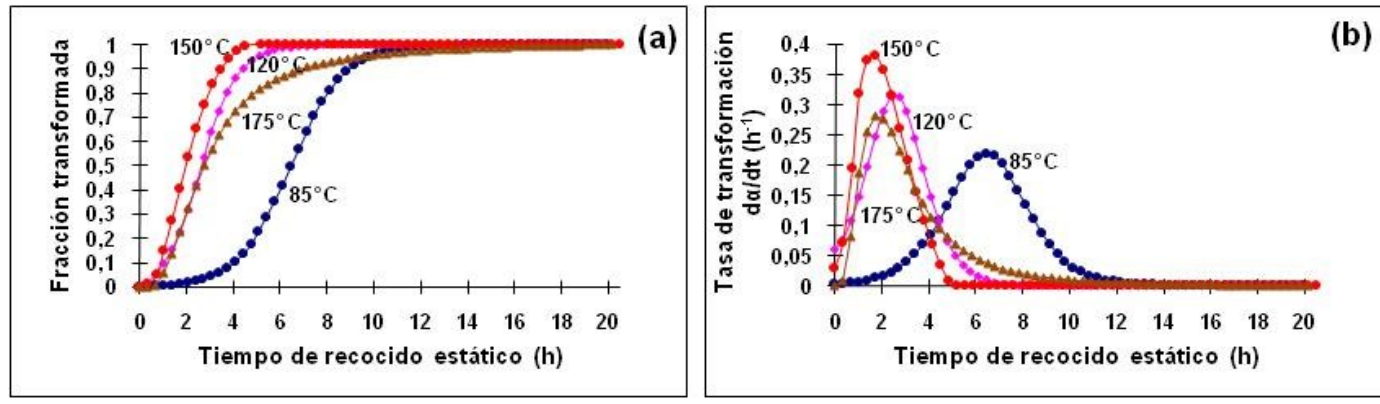

Figura 8: (a) Comportamiento de la fracción transformada, $\alpha$, en función del tiempo de recocido, a las temperaturas indicadas. (b) Comportamiento de la tasa de transformación, $\frac{\mathrm{d} \alpha}{\mathrm{dt}}$, en función del tiempo de recocido, a las temperaturas indicadas. Las líneas continuas que unen los puntos experimentales representan ajustes polinómicos.

En el gráfico de la Figura 8a, las curvas de la fracción transformada, $\alpha$, con base a la ecuación 1 y para las temperaturas de 85,120 y $150{ }^{\circ} \mathrm{C}$, muestran un perfil sigmoidal típico de un mecanismo de reacción dominante por control mixto: nucleación y crecimiento, gobernados por el fenómeno de difusión. La curva a $175^{\circ} \mathrm{C}$ muestra un perfil desacelerado, con mecanismos de reacción dominantes gobernados por difusión y crecimiento por intercaras [35-38].

\subsubsection{Modelo de KJMA-MITTEMEIJER y obtención de los parámetros cinéticos de la transformación}

En función de los gráficos de las Figuras (8a y 8b), una operación de transposición del eje de la fracción transformada, $\alpha$, en la Figura 8a, previa interpolación, al eje del tiempo de recocido (eje de las abscisas) en la Figura $8 \mathrm{~b}$, resulta en la obtención de un nuevo gráfico dado por la tasa de transformación, $\frac{\mathrm{d} \alpha}{\mathrm{dt}}$, en función de la fracción transformada, $\alpha$, este nuevo gráfico se muestra en las Figuras ( 9 a y 9b) para los puntos experimentales, con las temperaturas indicadas. Por otra parte, y seguidamente, la implementación y aplicación de la ecuación 7 a los puntos experimentales en los gráficos de las Figuras (9a y 9b), a las temperaturas indicadas, mediante un programa computacional de ajuste y optimización (programa de computador Microcal Origin versión 6), permite obtener los parámetros de ajuste, $\mathrm{n} \mathrm{y} \mathrm{k}$, y con ello las líneas continuas del ajuste teórico a los puntos experimentales, tal como se muestra en las Figuras ( $9 \mathrm{a}$ y $9 \mathrm{~b}$ ).
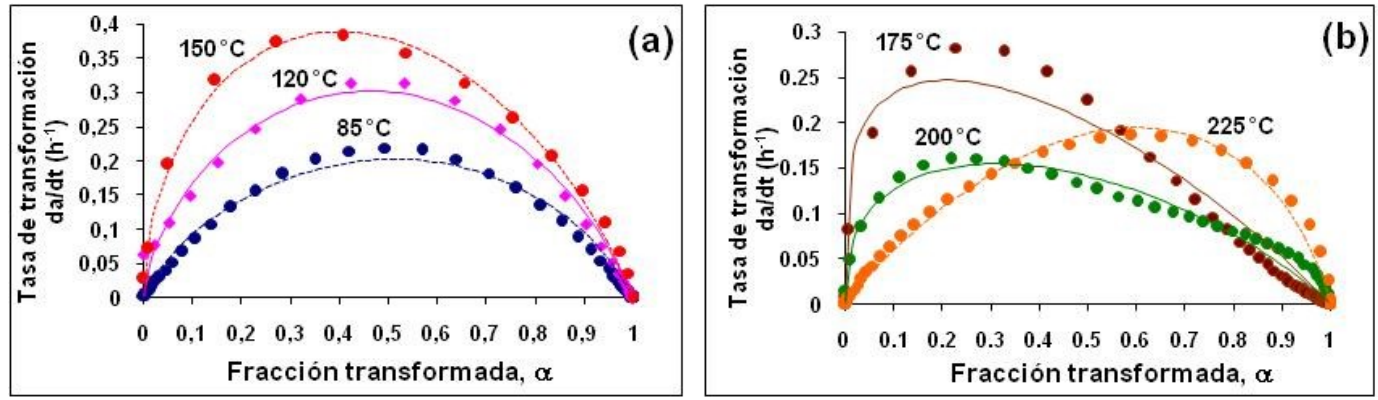
Figura 9: Tasa de transformación, $\frac{\mathrm{d} \alpha}{\mathrm{dt}}$, en función de la fracción transformada, $\alpha$. (a): temperaturas de 85,120 y $150{ }^{\circ} \mathrm{C}$. (b): temperaturas de 175,200 y $225^{\circ} \mathrm{C}$. Las líneas continuas corresponden al ajuste teórico (ecuación 7).

La Tabla 4 presenta los valores obtenidos para los parámetros cinéticos, $\mathrm{n}$ y $\mathrm{k}$, y sus respectivos estadísticos $\chi 2$ (ji o chi cuadrado: bondad del ajuste), y r2 (coeficiente de correlación lineal al cuadrado). Evaluar la bondad del ajuste es ver en qué medida se ajustan los datos observados a una distribución teórica esperada. $\chi 2$ contrasta las frecuencias observadas con las teóricas. El ajuste será más "bondadoso" en la medida en que $\chi 2$ muestre valores tendientes a cero, en este caso $\chi 2$ : 10-2 a 10-6. En el caso de r2, el ajuste será más correlacionado en la medida en que r2 se acerque al valor de la unidad, en este caso r2: 0,9492 a 0,9999. Estos valores indican una buena y "bondadosa" correlación entre los valores experimentales y el modelo aplicado.

Tabla 4: Parámetros cinéticos, $\mathrm{n}$ y $\mathrm{k}$, y sus estadísticos $\chi^{2} \mathrm{y} \mathrm{r}^{2}$ (bondad del ajuste). El parámetro $\tau$ representa el tiempo característico de transformación de fases.

\begin{tabular}{c|c|c|c|c|c}
\hline $\mathbf{T}\left({ }^{\circ} \mathbf{C}\right)$ & $\mathbf{n}$ & $\mathbf{k}\left(\mathbf{h}^{-1}\right)$ & $\chi^{\mathbf{2}}$ & $\mathbf{r}^{\mathbf{2}}$ & $\tau=\mathbf{1 / k}(\mathbf{h})$ \\
\hline 85 & 3,8224 & 0,1406 & $5,0 \mathrm{E}-5$ & 0,9997 & 7,1124 \\
\hline 120 & 2,2731 & 0,3293 & $2,0 \mathrm{E}-5$ & 0,9997 & 3,0367 \\
\hline 150 & 2,2086 & 0,4232 & $1,0 \mathrm{E}-5$ & 0,9999 & 2,3629 \\
\hline 175 & 1,5153 & 0,2718 & $7,4 \mathrm{E}-5$ & 0,9914 & 3,6792 \\
\hline 200 & 1,9165 & 0,1753 & $1,4 \mathrm{E}-5$ & 0,9989 & 5,7045 \\
\hline 225 & 4,6595 & 0,1071 & $1,0 \mathrm{E}-4$ & 0,9995 & 9,3371 \\
\hline 250 & 1,1605 & 0,3932 & $4,4 \mathrm{E}-6$ & 0,9999 & 2,5432 \\
\hline 275 & 10,8917 & 0,0704 & $1,6 \mathrm{E}-4$ & 0,9991 & 14,2045 \\
\hline 300 & 3,9073 & 0,0651 & $5,1 \mathrm{E}-3$ & 0,9492 & 15,3610 \\
\hline
\end{tabular}

Los valores obtenidos por el exponente de Avrami, n, hacen que este parámetro muestre un comportamiento parabólico entre las temperaturas de 85 y $225^{\circ} \mathrm{C}$, este tipo de comportamiento está asociado a una precipitación discontinua, preferencialmente en los bordes de grano, y cuyo mecanismo de reacción dominante es por control mixto: nucleación y crecimiento, y están gobernados por el fenómeno de difusión. Estos procesos promueven, al inicio, una gran diversidad de formas de crecimiento de dimensiones nanométricas, y con incremento en la tasa de nucleación; posteriormente el crecimiento de las partículas es de apreciable volumen con preferencial morfología acicular, argumentos similares fueron relacionados por CHRISTIAN J. W., [20]. El parámetro tasa de reacción, k, involucra la frecuencia a la cual se alcanza el estado activado [39]. Este parámetro, de acuerdo a sus valores, presenta un comportamiento oscilatorio amortiguado en función de las temperaturas de recocido estático consideradas. Por otro lado, usando el parámetro tasa de reacción (k) en la ecuación 3, y métodos gráficos, se determinó la energía de activación, $\mathrm{E}_{\mathrm{a}}$, $\mathrm{y}$ el factor de frecuencia de la tasa de reacción, $\mathrm{k}_{\mathrm{o}}$. Entre las temperaturas de 85 y $150{ }^{\circ} \mathrm{C}$, se obtuvo un valor de $22 \mathrm{~kJ} / \mathrm{mol}(0,24 \mathrm{eV} / \mathrm{at})$, que concuerda con el obtenido usando las medidas de resistividad no-isotérmica $\left(\mathbf{E}_{\mathbf{a}}^{\mathbf{I}}: \mathbf{2 0}-\mathbf{6 0} \mathbf{k J} / \mathbf{m o l}\right)$; y un valor para $\mathrm{k}_{\mathrm{o}} \approx 7 \times 10^{-2} \mathrm{~s}^{-1}$. Entre las temperaturas de 225 y $250{ }^{\circ} \mathrm{C}$ el valor de energía de activación obtenido fue de $113 \mathrm{~kJ} / \mathrm{mol}(1,22 \mathrm{eV} / \mathrm{at})$, de nuevo en correspondencia al obtenido previamente $\left(\mathbf{E}_{\mathbf{a}}^{\text {II: }} \mathbf{9 0}-\right.$ $120 \mathrm{~kJ} / \mathbf{m o l}$ ); y $\mathrm{k}_{\mathrm{o}} \approx 2 \times 10^{7} \mathrm{~s}^{-1}$. Estos valores de energías de activación son concordantes con los valores obtenidos por otros autores [26-32].

Considerando la aproximación atomística, y puesto que la tasa de reacción es equivalente al recíproco del tiempo de transformación de fases, $\tau$ (ver ecuación 3), por lo tanto, se puede determinar el Número de Deborah $\left(\mathrm{D}_{\mathrm{e}}\right.$; con $\tau \equiv \delta$ en la ecuación 8 ). La Figura 10a muestra el gráfico que representa el comportamiento del Número de Deborah en función de la temperatura de recocido estático, obtenido mediante la ecuación 8 , a partir del tiempo característico de transformación de fases (curva atomística), dados en la Tabla 4, (la línea continua que une los puntos experimentales representa un ajuste polinómico y muestra la tendencia seguida).

En función de esta curva, Figura 10a, y en concordancia con los postulados reológicos [16], se tiene: 1) Entre las temperaturas de 85 y $225^{\circ} \mathrm{C}$, se determinó un Número de Deborah en el intervalo $0,1 \leq \mathrm{D}_{\mathrm{e}} \leq 0,5$, asociado a un régimen de alta difusión, con una tasa de reacción máxima a $150{ }^{\circ} \mathrm{C}$ para un $\mathrm{D}_{\mathrm{e}} \geq 0,1$. 2) $\mathrm{A}$ temperaturas mayores a $225{ }^{\circ} \mathrm{C}$ se determinó el Número de Deborah en el intervalo $0,5 \leq \mathrm{D}_{\mathrm{e}} \leq 1$, asociado a un nivel de difusión medio con tendencia al decrecimiento hasta anularse. Por otro lado, la curva que representa la etapa de aceleramiento (también se muestra en la Figura 10a) exhibe un fuerte paralelismo y solapa- 
miento con la curva atomística (Figura 10a), esto indica que "el tiempo característico de transformación de fases, $\tau$ " es el equivalente porcentual al periodo de aceleramiento en los procesos de deformación, y de relajación de tensiones. Estos comportamientos están vinculados directamente con el comportamiento mostrado por la tasa de nucleación para la aleación de aluminio AA6061.

\subsubsection{Tasa de nucleación heterogénea}

En los sólidos la tasa de nucleación heterogénea [39-41], viene dada por la siguiente expresión:

$$
N_{h e t}^{\cdot}=v N_{v} e^{-\frac{\Delta G_{h e t}^{*}+E_{a}^{D}}{R T}}
$$

donde $v$ representa el factor frecuencia de vibración; $\mathrm{N}_{v}$ representa el número total de sitios de nucleación heterogénea por unidad de volumen; $\Delta G_{\text {het }}^{*}$ representa la barrera de energía contra la nucleación; $E_{a}^{D}$ representa la energía de activación para la difusión; $\mathrm{T}$ es la temperatura absoluta, y $\mathrm{R}$ la constante universal de los gases. Es bien sabido que durante las etapas tempranas de una reacción de precipitación la tasa de reacción es controlada por la tasa de nucleación, $\mathrm{N}_{\text {het }}^{*},[20,39-40]$, bajo tales condiciones, el tiempo, $\tau$, tomado para que precipite una cierta fracción de la nueva fase es inversamente proporcional a la tasa de nucleación:

$$
\mathrm{N}_{\text {het }}^{\cdot} \propto \frac{1}{\tau}
$$

así:

$$
\tau=\frac{\mathrm{c}_{1}}{\mathrm{~N}_{\mathrm{het}}}
$$

siendo $c_{1}$ una constante de proporcionalidad. En la Figura $10 \mathrm{~b}$ se muestra el gráfico que representa la tasa de nucleación heterogénea determinada a partir de la ecuación 9 en función de la temperatura de recocido estático para la aleación de alumínio AA6061 (la línea a trazos representa un ajuste polinómico y muestra la tendencia seguida). La curva sigue un comportamiento tipo gaussiano y alcanza un máximo a $150{ }^{\circ} \mathrm{C}$. Después de $250^{\circ} \mathrm{C}$ la tasa de nucleación decrece debido al agotamiento de los sitios de nucleación. Para la energía de activación para la difusión, $E_{a}^{D}$, se obtuvo un valor de $35 \mathrm{~kJ} / \mathrm{mol}(0,38 \mathrm{eV} / \mathrm{at}$.), este valor está dentro del intervalo de valores determinados previamente usando las medidas de resistividad eléctrica en regimen no-isotérmico $\left(\mathrm{E}_{\mathrm{a}}^{\mathrm{I}}: 20-60 \mathrm{~kJ} / \mathrm{mol}\right)[18]$.
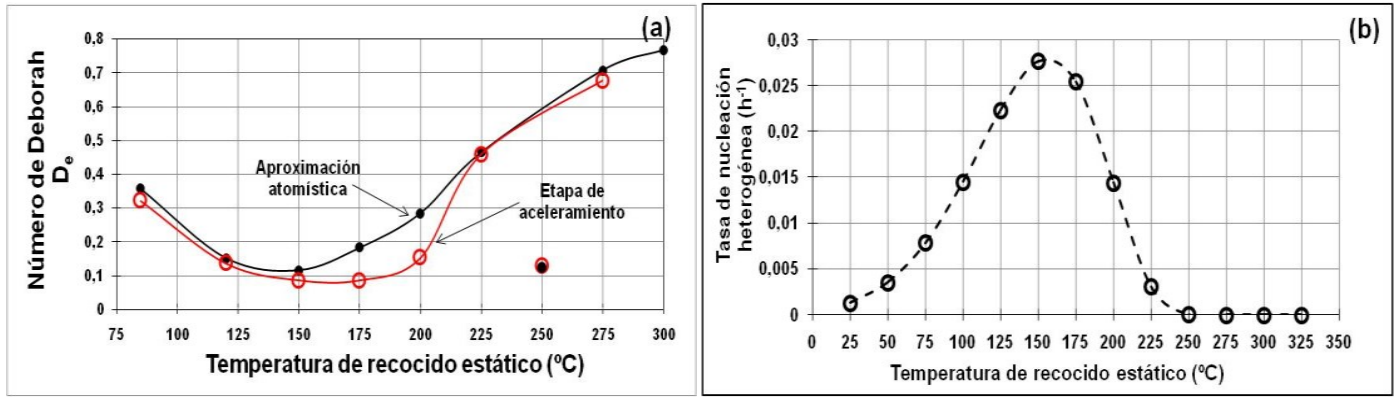

Figura 10: Aleación de alumínio AA6061 homogeneizada. (a) Comportamiento del Número de Deborah $\left(\mathrm{D}_{\mathrm{e}}\right)$ en función de la temperatura de recocido estático, a partir del "tiempo característico de transformación de fases, $\tau$ ", curva atómística (puntos sólidos), y la curva de aceleramiento ( $1^{\text {ra }}$ etapa, puntos abiertos, viene de la Figura 7a). (b) Tasa de nucleación heterogênea, $\mathrm{N}_{\text {het }}$, en función de la temperatura de recocido estático. Las líneas continuas y la línea a trazos que unen los puntos experimentales representan ajustes polinómicos y muestran las tendencias seguidas.

\section{CONCLUSIONES}

En la aleación de aluminio AA6061 el incremento de la temperatura, como acción dinámica, generó regiones de transformación de fases muy bien definidas. Y, como acción estática, reveló procesos de deformación y de relajación de tensiones. 
Cuatro regiones de transformación de fases fueron identificadas y caracterizadas: Región I: entre 20 y $190{ }^{\circ} \mathrm{C}$, con energías de activación, $\mathrm{E}_{\mathrm{a}}^{\mathrm{I}}: 20-60 \mathrm{~kJ} / \mathrm{mol}(0,22-0,66 \mathrm{eV} / \mathrm{at})$, asociadas con: energías de difusión de mono y di-vacancias, energías de migración y energías enlazantes de átomos de Mg y Si. El mecanismo de reacción dominante es por control mixto: nucleación y crecimiento, gobernados por difusión; con una tasa de reacción máxima a $150^{\circ} \mathrm{C}$. Estos comportamientos están vinculados directamente con las tasas de nucleación máximas registradas, con una energía de activación para la difusión, $\mathrm{E}_{\mathrm{a}}^{\mathrm{D}}=35 \mathrm{~kJ} / \mathrm{mol}(0.38$ eV/at). La MET reveló conglomerados de precipitados originados por la reubicación de los átomos de soluto, que conforman los agregados. Región II: entre 220 y $420{ }^{\circ} \mathrm{C}$, con un máximo a $350{ }^{\circ} \mathrm{C}$, y con energías de activación, $E_{\mathrm{a}}^{\mathrm{II}}: 90-120 \mathrm{~kJ} / \mathrm{mol}(0,99-1,32 \mathrm{eV} / \mathrm{at})$, asociadas con las energías para la difusión de $\mathrm{Mg}$ y Si en Al. La MET reveló precipitados aciculares mayores a $800 \mathrm{~nm}$, y correspondientes a las fases $\beta^{\prime \prime}$ y $\beta^{\prime}$, y embriones de $\beta$. Región III: entre 420 y $520^{\circ} \mathrm{C}$, con un máximo a $475^{\circ} \mathrm{C}$, y con $\mathrm{E}_{\mathrm{a}}^{\mathrm{III}}: 400-470 \mathrm{~kJ} / \mathrm{mol}(4,40-$ $5,17 \mathrm{eV} /$ at). La MET reveló precipitados cilíndricos mayores a $1000 \mathrm{~nm}$, y correspondientes a la fase $\beta\left(\mathrm{Mg}_{2} \mathrm{Si}\right)$. Región IV: A temperaturas mayores a $525^{\circ} \mathrm{C}$ se inicia la redisolución de las fases.

Los procesos de deformación y de relajación de tensiones, a nivel microestructural, presentaron etapas de aceleramiento, desaceleramiento y estacionariedad. Se verificó que existe un paralelismo y un solapamiento entre las curvas que representan estas etapas, en la aproximación macroestructural, con la curva que representa los procesos atomísticos de transformación de fases, en la aproximación microestructural. Se determinó un Número de Deborah, $\mathrm{D}_{\mathrm{e}} \leq 1$, y se asocia a un comportamiento reológico viscoelástico. Además, se observó que $\mathrm{D}_{\mathrm{e}}$ presenta un punto de inflexión alrededor de $150^{\circ} \mathrm{C}$ : durante la transformación ocurre un ablandamiento marcado por $0,260 \geq \mathrm{D}_{\mathrm{e}} \geq 0,085$, y después de la transformación ocurre un endurecimiento marcado por $0,085 \leq \mathrm{D}_{\mathrm{e}} \leq 0,920$. El tránsito experimentado por la aleación AA6061 de un ablandamiento (estado viscoso) a un endurecimiento (estado viscoelástico) convoca a considerar esta temperatura, $150{ }^{\circ} \mathrm{C}$, como un referencial tecnológico para procesos de endurecimiento de estas aleaciones de aluminio.

\section{AGRADECIMIENTOS}

El autor desea expresar su agradecimiento a la Dra. Marisel Diaz Baños por la revisión crítica del manuscrito. También expresa su agradecimiento al Instituto Venezolano de Investigaciones Científicas, IVIC, por el financiamiento para el desarrollo de este proyecto (Proyecto Semeruco 473-IVIC).

\section{BIBLIOGRAFÍA}

[1] ROJAS J. I., AGUIAR A., CRESPO D. "Effect of temperature and frequency of dynamic loading in the viscoelastic properties of aluminium alloy 7075-T6", Physica Status Solidi C, v. 8, n. 11-12, pp. 3111-3114, 2011.

[2] ROJAS J. I., CRESPO D. "Modeling of the Effect of Temperature, Frequency, and Phase Transformations on the Viscoelastic Properties of AA 7075-T6 and AA 2024-T3 Aluminum Alloys", Metallurgical and Materials Transactions A, v. 43, n. 12, pp., 2012.

[3] BALCH S. P., LAKES R. S. "The effects of composition, temperature, and annealing on the rheological properties of InZn in situ composites", Rheological Acta, v. 55, pp. 335-341, 2016.

[4] ASHBY M. F. "On the engineering properties of materials", Acta metallurgica, v. 37, n. 5, pp. 1273-1293, 1989.

[5] SCHALLER R., FANTOZZI G., GREMAUD G. (Editors), Materials Science Forum, v. 366-368, Mechanical Spectroscopy Q-1 2001: with Applications to Materials Science, Trans Tech Publications Ltd., Switzerland, ISBN 0-87849-876-1, pp. 3-684, 2001.

[6] MONDINO N., SCHOECK G. "Coherency Loss and Internal Friction", Physica status solidi A, v. 6, pp. 665-670, 1971.

[7] MONDINO, M. A., GUGELMEIER R. Proceedings of the 3rd European Conference on IFUAS, In: Smith, C. C. (Ed.), Pergamon, Manchester, pp. 317-320, 1980.

[8] SCHOECK G. "Fricción interna debido a la interacción entre dislocaciones y átomos soluto", Acta Metalurgica, v. 11, n. 6, pp. 617-622, 1963.

[9] SCHOECK G. "Internal Friction due to Precipitation", Physica Status Solidi, v. 38, pp. 651-658, 1969.

[10] SCHOECK G., BISOGNI E. "Internal Friction in Al-Ag Alloys", Physica Status Solidi, v. 32, pp. 31-40, 1969. 
[11] DIETER G. E., Jr. Metalurgia Mecánica, Editorial McGraw-Hill, Depósito Legal M 6889, Cap. 8, pp. 236-249, 1967.

[12] BLANTER M. S., GOLOVIN I. S., NEUHÄUSER H., et al. Internal Friction in Metallic Materials, A Handbook, Springer-Verlag, Berlin Heidelberg, ISSN 0933-033X, ISBN-10 3-540-68757-2, Cap. 3, pp. 121156, 2007.

[13] HYUN S., HOOGHAN T. K., BROWN W. L., et al. "Linear viscoelasticity in aluminum thin films", APPLIED PHYSICS LETTERS, v. 87, n. 061902, pp. 1-3, 2005.

[14] MITTEMEIJER E. J. "Review: Analysis of the kinetics of phase transformations", Journal of Materials Science, v. 27, pp. 3977-3987, 1992.

[15] MITTEMEIJER E. J. Fundamentals of Materials Science: The Microstructure-Property Relationship Using Metals as Model Systems, Springer-Verlag, Berlin Heidelberg, Cap. 7, pp. 303-337, ibid pp. 371-461, 2010.

[16] REINER M. “The Deborah Number", Physics Today, v. 17, n.1, p. 62, 1964.

[17] SIMMONS R. O., BALLUFFI, R. W. "Measurements of the high-temperature electrical resistance of aluminum: resistivity of lattice vacancies", Physical Review, v. 117, pp. 62-68, 1960.

[18] OCHOA-M., J. L. Estudio del Fenómeno de Precipitación en la Aleación de Aluminio AA6061 Mediante Medidas de Resistividad Eléctrica, Calorimetría y Técnicas Microscópicas, Tesis de D.Sc., Universidad Central de Venezuela, Caracas - Venezuela, 2010.

[19] OCHOA-M., J. L., GONZÁLEZ, G., LOZADA, L. "Transformación microestructural in situ y su correlación con la resistividad eléctrica en la aleación de aluminio AA6061", Revista Latinoamericana de Metalurgia y Materiales, v. 32, n. 2, pp. 185-194, 2012.

[20] CHRISTIAN J. W. The Theory of Transformations in Metals and Alloys., Part I: Equilibrium and General Kinetic Theory, Second Edition, Pergamon Press, pp. 1-20, ibid pp. 525-556, 1975.

[21] OCHOA-M., J. L. "Interpretación de las transformaciones de fase en régimen no-isotérmico en el aluminio AA6061 mediante el modelo termocinético de Mittemeijer: Parte II", Acta Microscopica, v. 24, n. 2, pp. $118-137,2015$.

[22] ŠESTÁK J., BERGGREN G. "Study of kinetics of the mechanism of solid-state reactions at increasing temperatures", Thermochimica Acta, v. 3, pp. 1-12, 1971.

[23] BABAK V. G., DESBRIÉRES J. "Deborah number for the viscoelasticity of adsorption layers of amphiphilic polyelectrolytes", Mendeleev Commun, v. 15, n. 5, pp. 190-191, 2005.

[24] MINFORD J. D. Handbook of Aluminum Bonding Technology and Data, Marcel Dekker, INC., ISBN: 0-8247-8817-6, 1993.

[25] OCHOA-M., J. L., OCHOA J., GONZÁLEZ G. "Influencia de la velocidad de calentamiento en la cinética de precipitación en el aluminio AA6061 a través de medidas de resistividad eléctrica", Revista de la Facultad de Ingeniería de la UCV, v. 23, n. 3, pp. 17-26, 2008.

[26] ESMAEILI S., POOLE W. J., LLOYD D. J. "Electrical resistivity studies on the precipitation behaviour of AA-6111”, Materials Science Forum, v. 331-337, pp. 995-1000, 2000.

[27] TAWFIK N. L., ABDEL H. E. M., KASSEM N. E. "Hardness and Electrical Resistivity Changes during Ageing of Rapidly Solidified Al-12.5Si-1Mg Alloy", Materials Transactions, JIM, v. 38, n. 5, pp. 401-405, 1997.

[28] MONDOLFO L. F. Aluminum Alloys: Structure and Properties, Butterworth \& Co. Publishers, Ltd., London-Boston, ISBN 040870680 5, Part 1, pp. 1-210, 1976.

[29] KORNGIEBEL E., LÖFFLER H., OETTEL W. "On the kinetics of the decomposition of Al-Mg-Si alloys", Physica Status Solidi (a), v. 30, n. 2, pp. K125-K127, 1975.

[30] CHATTERJEE D. K., ENTWISTLE K. M. "A study of effect of magnesium loss and of the addition of copper on the ageing of aluminium-magnesium-silicon alloys", Journal of the Institute of Metals, v. 101, pp. 53-59, 1973.

[31] KOVÁCS I., LENDVAI J., NAGY E. "The Mechanism of Clustering in Supersaturated Solid Solutions of Al-Mg2Si Alloys", Acta Metallurgica., v. 20, n. 7, pp. 975-983, 1972.

[32] PANSERI C., FEDERIGHI T. "A Resistometric Study of Preprecipitation in an Aluminium-1.4\% Mg2Si alloy", Journal of the Institute of Metals, v. 94, pp. 99-107, 1966. 
[33] KÊ T. S. "Experimental Evidence of the Viscous Behavior of Grain Boundaries in Metals", Physical Review, v. 71, n. 8, pp. 533-546, 1947.

[34] ABRIKOSOV A. A. Fundamentals of the Theory of Metals, Elsevier Science Publishers B. V., Netherlands, Cap. XX, pp. 33-46, 1988.

[35] VYAZOVKIN S., BURNHAM A. K., CRIADO J. M., et al., "ICTAC Kinetics Committee recommendations for performing kinetic computations on thermal analysis data", Thermochimica Acta, v. 520, pp. 1-19, 2011.

[36] PÉREZ-MAQUEDA L. A., CRIADO J. M., SÁNCHEZ-JIMÉNEZ P. E. "Combined Kinetic Analysis of Solid-State Reactions: A Powerful Tool for the Simultaneous Determination of Kinetic Parameters and the Kinetic Model without Previous Assumptions on the Reaction Mechanism", The Journal of Physical Chemis$\operatorname{try} A$, v. 110, pp. 12456-12462, 2006.

[37] KHAWAM A., FLANAGAN D. R. "Solid-State Kinetic Models: Basics and Mathematical Fundamentals", The Journal of Physical Chemistry B, v. 110, pp. 17315-17328, 2006.

[38] GALWEY A. K., BROWN M. E. "Kinetic background to thermal analysis and calorimetry", Handbook of Thermal Analysis and Calorimetry. v.1: Principles and Practice, Elsevier Science B.V., First edition 1998, Second impression 2003, ISBN: 0-444-82085-X, chap. 3, pp. 147-224, 2003.

[39] PORTER D. A., EASTERLING K. E. Phase Transformations in Metals and Alloys, Published by Van Nostrand Reinhold Company, New York, Cap.1, pp. 1-59., cap. 5, pp. 263-381, 1981.

[40] MORRIS E. F. Introduction to Phase Transformations in Condensed Systems, Second Printing, New York, Macmillan Series in Materials Science, cap.2, pp. 7-46, 1965.

[41] BRATLAND D. H., GRONG O., SHERCLIFF H., et al. "Modelling of Precipitation Reactions in Industrial Processing", Acta Materialia, v. 45, pp. 1-22, 1997. 